\title{
Mapping Electrochemical Heterogeneity at Gold Surfaces: A Second Harmonic Imaging Study
}

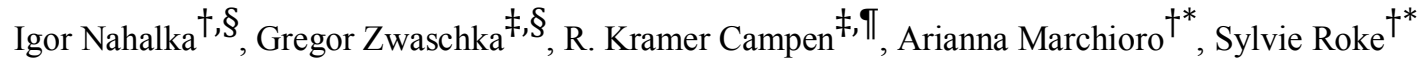 \\ † Laboratory for fundamental BioPhotonics (LBP), Institute of Bio-engineering (IBI), and Institute of
} Materials Science (IMX), School of Engineering (STI), and Lausanne Centre for Ultrafast Science (LACUS), École Polytechnique Fédérale de Lausanne (EPFL), CH-1015, Lausanne, Switzerland, f Fritz Haber Institute of the Max Planck Society, Faradayweg 4-6, 14195 Berlin, Germany

I Faculty of Physics, University of Duisburg-Essen, Lotharstraße 1, 47057 Duisburg, Germany

$\S$ Both authors contributed equally to this work. *E-mail correspondence: Arianna.marchioro@epfl.ch,sylvie.roke@epfl.ch

\begin{abstract}
Designing efficient catalysts requires correlating surface structure and local chemical composition with reactivity on length scales from nanometers to tens of microns. While much work has been done on this structure/function correlation on single crystals, comparatively little has been done for catalysts of relevance in applications. Such materials are typically highly heterogeneous and thus require methods that allow mapping of the structure/function relationship during electrochemical conversion. Here, we use optical second harmonic imaging combined with cyclic voltammetry to map the surface of gold nanocrystalline and polycrystalline electrodes during electrooxidation and to quantify the spatial extent of surface reconstruction during potential cycling. The wide-field configuration of our microscope allows for real-time imaging of an area $\sim 100 \mu \mathrm{m}$ in diameter with submicron resolution. By analyzing the voltage dependence of each pixel, we uncover the heterogeneity of the second harmonic signal and quantify the fraction of domains where it follows a positive quadratic dependence with increasing bias. There, the second harmonic intensity is mainly ascribed to electronic polarization contributions at the metal/electrolyte interface. Additionally, we locate areas where the second harmonic signal follows a negative quadratic dependence with increasing bias, which also show the largest changes during successive cyclic voltammetry sweeps as determined by an additional correlation coefficient analysis. We assign these areas to domains of higher roughness that are prone to potential-induced surface restructuring, and where anion adsorption occurs at lower potentials than expected based on the $\mathrm{CV}$.
\end{abstract}




\section{Introduction}

The surface structure of a catalyst has a tremendous impact on its activity. ${ }^{1-3}$ Therefore, revealing catalysts surface heterogeneity is of major importance. This is particularly true for catalysis at the liquid/solid interface, which is essential for many energy conversion and storage processes, e.g., water electrolysis. Much past work has tried to establish the relationship between surface structure and activity. Such efforts are challenging for a variety of reasons, as for example: (1) The presence of defects or grain boundaries can significantly alter the local reactivity by changing the surface charge density or the local conductivity, ${ }^{4,5}(2)$ The surface strain of metal catalysts has also been demonstrated to be correlated with catalytic activity ${ }^{6,7}$ and (3) restructuring of the surface under operating conditions can also contribute to changes in local activity. ${ }^{8-10}$ Designing an efficient catalyst thus requires correlating surface structure and the nature of the catalytically active sites with activity on submicron length scales under operating conditions. For electrocatalysis this challenge can be addressed by experimental techniques that collect both the electrochemical response and the topography/local chemical composition $o p$ erando.

Several techniques can be used to probe topography, local activity and compositional spatial heterogeneity under operating conditions. Electrochemical atomic force microscopy (EC-AFM) can be used to investigate changes in electrode morphology during an electrochemical reaction, with a resolution in the $\mathrm{nm}$ to $\mu \mathrm{m}$ range. ${ }^{11,12}$ Scanning electrochemical microscopy (SECM) and variations of this technique have proven very valuable in the study of electrochemical processes at various interfaces, by probing both the topography and the local reactivity with sub- $\mu \mathrm{m}$ spatial resolution. ${ }^{13,14}$ It has been recently shown that electrochemical scanning tunneling microscopy (EC-STM) can map the catalytic activity of surfaces with sub-nm to nm spatial resolution by comparison of local fluctuations in the tunneling current above reactive and non-reactive nanoscale surface sites. ${ }^{15}$ Chemical specificity can be obtained by electrochemical tip-enhanced Raman spectroscopy (EC-TERS), which shows great promise to map active-site chemistry with a precision of $10 \mathrm{~nm}$ by combining Raman spectroscopy with ECSTM. ${ }^{16}$ However, none of these techniques would be suitable to study systems where electrochemical reactions produce, for example, gas bubbles. In addition to this limitation, all these techniques rely on a rastering scheme, where the signal is collected pixel by pixel. This scheme implies a time delay in collecting the signal from different areas, which prevents monitoring of large-scale changes in real time. It also becomes time-consuming if applied to large surface areas (in the hundreds of $\mu \mathrm{m}$ range), which can be a drawback in an application-oriented context where large-scale screening of samples is needed. As all practical (photo)electrocatalysts are extremely heterogeneous due to their large surface areas, the ability of large-scale screening of the surface is desirable to localize the presence of "hot spots" of increased reactivity. 
Imaging of surfaces in real time (i.e., without employing a rastering scheme) can be obtained by high-throughput wide-field second harmonic (SH) microscopy. We have recently reported a study of the silica/water interface where we imaged the interfacial structure and dynamics of water in a microscopically confined geometry, using a structurally-illuminated $\mathrm{SH}$ wide-field microscope.$^{17}$ Dissociation constants for the silica surface deprotonation could be extracted from surface potential maps employing a suitable model relating surface potential, charge and chemistry, and were found to change over 9 orders of magnitude along the surface of a single capillary because of surface heterogeneity. Contrarily to conventional rastering SH microscopes, here the wide-field configuration enables the imaging of spatial heterogeneity in real-time for areas of $100 \mu \mathrm{m}$ in diameter and enables imaging of planar surfaces with detection along the surface normal. Furthermore, the imaging throughput is improved by 2-3 orders of magnitude compared to a point-scan imaging, ${ }^{18}$ bringing the image acquisition time down to $\mathrm{ms}^{17}$ or even $\mu \mathrm{s} .{ }^{19,20}$ Such a short acquisition time is extremely valuable for dynamical imaging, ${ }^{17}$ where surface changes could be monitored with $\mu$ s to ms time resolution.

The advantages of this wide-field microscopy configuration are further combined with second-harmonic generation (SHG), which has been used to study the electrode-electrolyte interface for decades. ${ }^{21,22,31,23-30} \mathrm{SHG}$ is an optical, non-invasive and label-free technique and only requires a broken centrosymmetry of the media to generate a signal, making it interface-specific. $^{32}$ This requirement makes SH particularly suitable for studying centrosymmetric metal electrodes such as gold or silver, where the SH signal mainly arises from the broken symmetry at the electrode/electrolyte interface. In these experiments there are a number of contrast mechanisms: The SH light response is complex and influenced by many factors. For metals, the SH primarily originates from the charge gradient at the interface and is consequently sensitive to modifications of this gradient. The applied voltage modifies the surface charge density at the interface and therefore the SH intensity. At sufficiently positive potentials, the formation of oxide layers on the surface will then start to quench the SH signal by reducing the free charge density. ${ }^{31,33,34}$ This quenching is particularly strong if the SH signal is resonant with a surface plasmon associated with d-electrons, as this contribution disappears upon oxidation. ${ }^{31}$ Besides the exposed surface structure (for example (111) vs. (110)), ${ }^{24,35}$ ion sorption (adsorption and desorption), ${ }^{34,36}$ surface reconstruction, ${ }^{26,34,37}$ and water orientation in the adjacent electrolyte solution ${ }^{38}$ can all contribute to the $\mathrm{SH}$ response and are all a function of surface structure. Lastly, illumination angle ${ }^{39}$ wavelength, as well as SH wavelength have to be considered: ${ }^{26,33,40-42} \mathrm{~A}$ resonance with surface plasmons, either due to a free-standing electron wave around $550 \mathrm{~nm}$ or associated with d-electrons in the regions of 410 and $270-280 \mathrm{~nm},{ }^{43}$ will influence the SH intensity. While it is difficult to disentangle all the contributions to the SH response, it is possible reduce their number by carefully selecting the experimental parameters. 
In this paper, we use wide-field high-throughput $\mathrm{SH}$ imaging combined with cyclic voltammetry $(\mathrm{CV})$ to study the spatial extent of gold surface chemistry on nanocrystalline and polycrystalline electrodes during metal electrooxidation. Understanding metal electrooxidation is desirable, because it precedes the oxygen evolution reaction (OER), the oxidative half-cell reaction of water electrolysis. The OER is performance-limiting in virtually every electrolyser ${ }^{44}$ and finding better catalysts for it is (among other factors) complicated by our lacking understanding of the oxidation process and the resulting oxide. Using two different types of image analyses and without any a priori knowledge of the surface structure, we show that the SH response as a function of applied potential in polycrystalline electrodes displays strong heterogeneity on the order of $\mu \mathrm{m}$ as opposed to the nanocrystalline electrodes with sizes of single grains below the imaging resolution, which show a uniform $\mathrm{SH}$ vs. potential profile across the entire field of view. Analyzing the voltage dependence of each pixel on the polycrystalline electrode, we identify areas on the sample where the SH signal follows a positive quadratic dependence with increasing bias. There, the SH intensity is mainly attributed to electronic polarization contributions at the metal/electrolyte interface. Additionally, we identify areas where the SH signal follows a negative quadratic dependence with increasing bias. These latter sites correspond to areas that show the largest changes in successive CV sweeps, as corroborated by a correlation coefficient analysis. We assign these areas to domains of higher roughness that show an anion adsorption behavior distinctively different from the average and are more prone to potential-induced surface reconstruction. Rough and dynamic surfaces are often correlated to catalytic activity. ${ }^{45,46}$ Lastly, we show that those same areas appear as the brightest in the PSS polarization combination for our experimental conditions.

\section{Materials and Methods}

\section{Chemicals}

Sodium phosphate monobasic monohydrate ( $\geq 99.0 \%$ purity, Sigma-Aldrich), hydrogen peroxide (30\% Reactolab SA), and sulfuric acid (95-97\%, ISO, Merck) were used as received. All aqueous solutions were made with ultrapure water $\left(\mathrm{H}_{2} \mathrm{O}\right.$, Milli-Q UF plus, Millipore, Inc., resistivity of $18.2 \mathrm{M} \Omega \cdot \mathrm{cm})$.

\section{Electrochemistry}

A homebuilt Teflon cell was used for the microscopy experiments under potential control. The cell was cleaned by immersion in Piranha solution overnight and repeated supersonication in Milli-Q water before the experiment. Details of the cleaning procedure can be found in our previous work. ${ }^{47}$ As shown in Fig. 1A, the gold electrode is sandwiched between the substrate borofloat ${ }^{\circledR}$ glass and PTFE cell. The phosphate buffer solution $\left(\mathrm{NaH}_{2} \mathrm{PO}_{4}\right)$ of $\mathrm{pH}=2.8$ is on top of the gold electrode immersing the microscope objective, the platinum counter electrode and the reversible hydrogen reference electrode. The absence of traces of chloride and sulfate 
ions was cross-checked in separate experiments where we intentionally introduced those contaminants on the gold electrode (See Supporting Information), which showed that our $0.5 \mathrm{M}$ $\mathrm{NaH}_{2} \mathrm{PO}_{4}$ electrolyte is not contaminated with chloride or sulfate ions. The polycrystalline gold foils were purchased from Sigma-Aldrich ( $0.025 \mathrm{~mm}$ thickness, $99.99 \%$ trace-metal basis) and annealed at $500{ }^{\circ} \mathrm{C}$ for $2 \mathrm{~h}$ (with linear heating and cooling ramps) to achieve a grain size distribution characteristic of many practically relevant catalysts and suitable for our microscope. The nanocrystalline gold thin-film sample was obtained by physical vapor deposition of 200 $\mathrm{nm}$ of gold on an optically flat glass substrate with a $30 \AA$ layer of $\mathrm{Cr}$ for increased adhesion. Before use, both electrodes were cleaned using the following procedure: copiously rinsed in acetone, copiously rinsed with Milli-Q water, exposed to ozone for $5 \mathrm{~min}$ in a UV ozonator, copiously rinsed with Milli-Q water, copiously rinsed with electrolyte, and annealed electrochemically until the CV was stable (generally 20-30 cycles of cyclic voltammetry). After this procedure, no organic contamination is apparent in the $\mathrm{CV}$. The counter electrode was a $\mathrm{Pt}$ mesh, which was cleaned identically to the Teflon cell. A self-made reversible hydrogen electrode was employed as reference. ${ }^{48}$ The potential cycling ranged from 0 to $1.7 \mathrm{~V}$ vs. the reversible hydrogen electrode (RHE) for the nanocrystalline gold film; and from 0.1 to $1.6 \mathrm{~V}$ vs. RHE for the polycrystalline gold foil.

\section{Imaging experiments}

The SH imaging setup has been previously described in detail for its transmission geometry ${ }^{17}$ and is constructed using principles from SH scattering. ${ }^{49}$ Here, we use the one-beam reflection geometry displayed in Fig. 1B. The light source for the SH microscope is a Pharos SP-1.5 (Light Conversion), which delivers $180 \mathrm{fs}$ pulses centered around $1030 \mathrm{~nm}$ at a maximum output power of $6 \mathrm{~W}$ and variable repetition rates between $1 \mathrm{kHz}-1 \mathrm{MHz}$. The laser system is operated at a repetition rate of $200 \mathrm{kHz}$. Half of the source intensity is used in our experiment. The fundamental beam is sent to a spatial light modulator (SLM). The SLM (Holoeye Pluto-NIR015 ) is a phase-only device coated for near-infrared wavelengths. We project a binary grating on the SLM that acts as a reflective diffraction grating. The laser light is reflected towards a polarization state generator composed of a quarter-wave plate followed by a half-wave plate to modify the polarization state of the laser light. Diffraction orders are then formed in the Fourier plane, where we block all the orders except one $1^{\text {st }}$ order. The laser light propagates through additional lenses and mirrors, until it is reflected by a dichroic mirror and focused into the backfocal plane of a water immersion microscope objective (Olympus, LUMPFLN 60XW, NA = $1.0, \mathrm{WD}=2 \mathrm{~mm}$ ). This ensures that the sample is illuminated with a collimated wide-field single beam at $34^{\circ}$ with respect to the surface normal. The gaussian illumination at the sample has a diameter of $82 \mu \mathrm{m}$ full-width at half maximum with peak power density of $17.1 \mathrm{GW} / \mathrm{cm}^{2}$ ( $3.4 \mathrm{~mJ} / \mathrm{cm}^{2}$ fluence). The position of the sample is manipulated by a XYZ translation stage 
(Asi Imaging, PZ-2000), where the XY-axes are controlled by actuators with $10 \mathrm{~cm}$ travel range, while the $Z$-axis is moved by a piezoelectric stage with $300 \mu \mathrm{m}$ travel range. As a complementary feature of this positioning system, the microscope objective is mounted on a Z-axis actuator stage (Asi Imaging, LS-200). After the SH photons are generated at the surface of the sample, they propagate mainly in the direction of the reflected laser light. The reflected SH photons further pass through a dichroic mirror and continue towards a short-pass and a bandpass filter to ensure that we detect only SH photons at the camera. The polarization state of the SH light is analyzed with a half-wave plate followed by a polarizing beam splitter. Afterward, the SH photons are detected by a back-illuminated electron-multiplied and intensified CCD camera with 512x512 pixels (PI-MAX4: 512EM-HBf P46 GEN III). The size of a single pixel corresponds to $\sim 400 \mathrm{x} 400 \mathrm{~nm}$ in the sample plane. All microscope mirrors on the path are protected silver mirrors (Thorlabs, PF10-03-P01). The lenses (achromatic) and the other optical elements on the laser (illumination) path $(1030 \mathrm{~nm})$ are near-infrared antireflection coated (Thorlabs, B), while the optical elements on the SH (detection) path $(515 \mathrm{~nm})$ are antireflection coated for the visible region (Thorlabs, A). All SH videos were acquired with $0.25 \mathrm{~s}$ acquisition time per frame either in PPP, or PSS polarization combinations, where the first letter stands for the polarization state of the SH light and the last two letters stand for the polarization state of the two photons of the fundamental beam illuminating the sample. We chose here a slower acquisition in order to have an optimum CV shape, however, it is possible to decrease the acquisition time down to $10 \mathrm{~ms}$ while still retaining an optimum SH signal-to-noise ratio. Further illustration of the sample illumination can be found in the supplementary information.

A

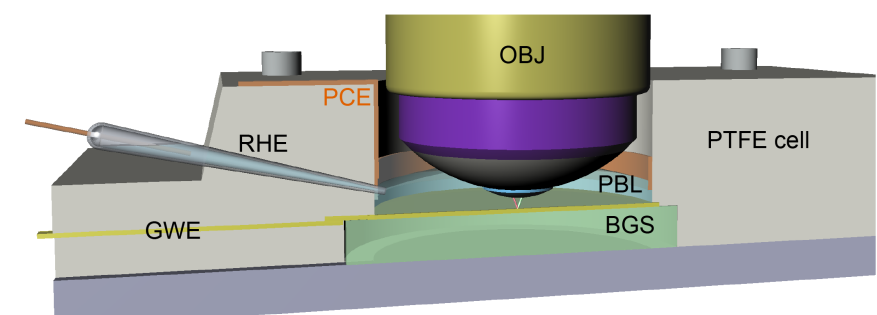

B

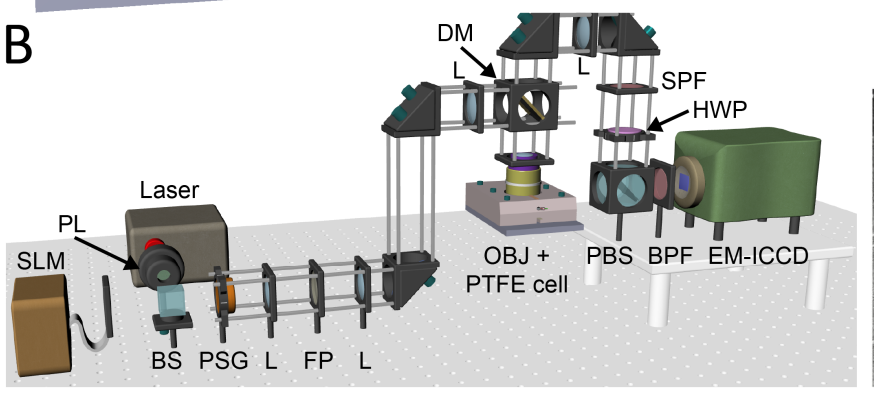

C

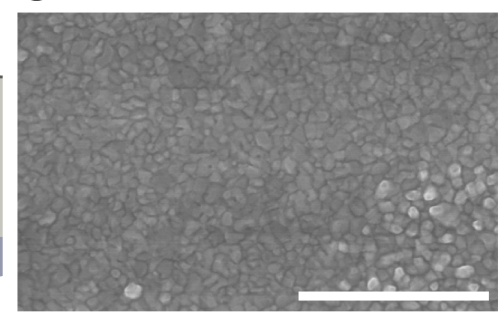

D

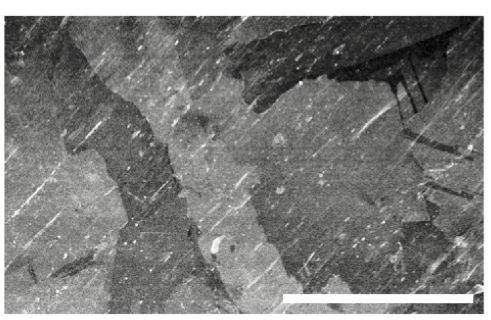

Figure 1: Experimental setup and samples. (A) PTFE cell for SH and CV measurements; GWE - gold working electrode, RHE - reversible hydrogen electrode, PCE - platinum counter electrode, PBL - phosphate buffer liquid, BGS - borofloat ${ }^{\circledR}$ glass substrate. (B) Schematic of the second harmonic microscope in reflection mode of operation; SLM - spatial light modulator, PL - polarizer, BS - beam splitter, PSG - polarization state generator, L - 
achromatic lens, FP - Fourier plane, DM - dichroic mirror, OBJ - microscope objective, SPF - short pass filter, HWP - half wave plate, PBS - polarizing BS, BPF - second harmonic band-pass filter, EM-ICCD - electron multiplying gated camera. (C), (D) Gold nanocrystalline and polycrystalline samples imaged by scanning electron microscopy, respectively.

\section{Results \& Discussions}

Before discussing the data, we briefly summarize the main elements of the model pioneered by Lee et al. ${ }^{50}$ that we use here below to analyze the SH images. Lee et al. found that, under certain conditions, the SH intensity produced in reflection on silicon and silver surfaces could be described as a quadratic function of the applied potential, and that the surface susceptibility varied linearly with the applied potential. This was further validated by later work..$^{22,33,40}$ The nonlinear polarization can be described as the sum of dipolar electric polarizations and static dc-fieldinduced polarizations:

$$
P(2 \omega)=\chi^{(2)}(2 \omega) E(\omega) E(\omega)+\chi^{(3)^{\prime}}(2 \omega) E(\omega) E(\omega) E_{D C},
$$

where $\chi^{(2)}$ is the total second-order nonlinear susceptibility from both surface and bulk (for gold, the surface term dominates over the bulk term $)^{51}$ and $\chi^{(3)^{\prime}}$ is the effective total third-order nonlinear susceptibility. The effective third-order nonlinear susceptibility includes both the material response as well as the contribution of all oriented dipoles (mainly solvent) in the solution. In the case of gold, the dipole response is much smaller than the gold response. $E(\omega)$ is the electric field associated with the fundamental laser beam, and $E_{D C}$ is a static electric field across the interface. The intensity of the SH signal $I(2 \omega)$ can then be written as proportional to:

$$
I(2 \omega) \sim\left|\chi^{(2)}+\chi^{(3)} \Phi_{0}\right|^{2} I(\omega)^{2}
$$

where $\Phi_{0}$ is the potential drop across the metal surface (the difference in potential between the gold surface and bulk electrolyte), and $I(\omega)$ is the intensity of the fundamental beam. ${ }^{33}$ Here, $\chi^{(2)}$ and $\chi^{(3)^{\prime}}$ are assumed to be potential-independent. This can be reduced to a parabolic equation:

$$
I(2 \omega) \sim\left(\chi^{(3)^{\prime}} \chi^{(3)^{* *}}\right) \Phi_{0}^{2}+\left(\chi^{(2)} \chi^{(3)^{* *}}+\chi^{(2)^{*}} \chi^{(3)^{\prime}}\right) \Phi_{0}+\left(\chi^{(2)} \chi^{(2)^{*}}\right)=Q \Phi_{0}^{2}+L \Phi_{0}+C,
$$

where $Q, L$, and $C$ are the quadratic, linear, and constant coefficient, respectively. We assume that the potential drop at the metal surface is:

$$
\Phi_{0}=V-V_{P Z C},
$$


where $V$ is the applied potential and $V_{P Z C}$ is the potential of zero charge. Using Eqs. (3) and (4), one can express the ratio of $\mathrm{Q} / \mathrm{L}$ as follows:

$$
\frac{Q}{L}=\frac{\chi^{(3)^{\prime}} \chi^{(3)^{* *}}}{\chi^{(2)} \chi^{(3)^{* *}}+\chi^{(2)^{*}} \chi^{(3)^{\prime}}}=\frac{\operatorname{Re}\left(\chi^{(3)^{\prime}}\right)^{2}+\operatorname{Im}\left(\chi^{(3)^{\prime}}\right)^{2}}{2 \operatorname{Re}\left(\chi^{(2)}\right) \operatorname{Re}\left(\chi^{(3)^{\prime}}\right)+2 \operatorname{Im}\left(\chi^{(2)}\right) \operatorname{Re}\left(\chi^{(3)^{\prime}}\right)} .
$$

According to the experimental data reported in the literature, ${ }^{52-55}$ the ratio of $\operatorname{Re}\left(\chi^{(3)}\right) / \operatorname{Im}\left(\chi^{(3)}\right)$ increases with increasing fundamental wavelength. As a consequence, the imaginary part can be neglected for fundamental wavelengths in the NIR, as already done in a previous study using $1060 \mathrm{~nm}$ fundamental wavelength (close to the fundamental wavelength we use in this work, $1030 \mathrm{~nm}) .{ }^{40}$ We additionally performed a cross-check using Miller's rule, which also confirmed the relatively small value of $\operatorname{Im}\left(\chi^{(3)}\right)$ (see Supplementary Information). This simplifies Eq. (3) to:

$$
\frac{Q}{L} \sim \frac{\operatorname{Re}\left(\chi^{(3)^{\prime}}\right)}{\operatorname{Re}\left(\chi^{(2)}\right)}
$$

For simplicity, from now on we will omit the real part notation and refer to $\operatorname{Re}\left(\chi^{(3)}\right)$ and $\operatorname{Re}($ $\left.\chi^{(2)}\right)$ in the rest of the manuscript as $\chi^{(3)^{\prime}}, \chi^{(2)}$, respectively. If $\chi^{(3)^{\prime}} \gg \chi^{(2)}$, then the signal is parabolic with a minimum at the $V_{P Z C}$. Conversely, if $\chi^{(3)} \ll \chi^{(2)}$, the signal is no longer parabolic and dominated by second-order contributions of surface and bulk. If $\chi^{(3)^{\prime}} \approx \chi^{(2)}$ then the signal is parabolic with a minimum shifted away from the $V_{P Z C}$. It is important to note that the assumptions in Equations (2) and (4) are only valid in the absence of specifically adsorbing ions, or in a potential region where no specific ion adsorption occurs, i.e., when tuning the electrode potential is analog to changing the charge density on a bare metal capacitor. Additionally, Guyot-Sionnest et al. ${ }^{33}$ have shown that for fundamental wavelengths with photon energies within the free-electron regime the third-order contribution is important, while this contribution becomes negligible for photon energies that allow for interband transitions (for gold, this corresponds to wavelengths below $\sim 500 \mathrm{~nm}^{56}$ ). In the interband transition regime, the second order susceptibility is predominant, and the variation of the $\mathrm{SH}$ signal with potential is likely correlated to the screening of the d-electron contribution by the surface free electron distribution. ${ }^{33}$

\section{SH imaging of nanocrystalline and polycrystalline electrodes}

In Fig. 2, the surface of a nanocrystalline gold electrode is imaged with the SH microscope in a phosphate buffer electrolyte $\left(0.5 \mathrm{M} \mathrm{NaH}_{2} \mathrm{PO}_{4}, \mathrm{pH}=2.8\right)$ while performing $\mathrm{CV}$. Phosphate ions were chosen because of their rather low propensity to adsorb on the surface ${ }^{57}$ and to ensure 
$\mathrm{pH}$ stability during measurements. During potential cycling, the increasing of potential towards positive values is referred to as the forward scan, and the decreasing of potential towards negative values as the reverse.

Fig. 2A displays an SH image taken at $1.2 \mathrm{~V}$ during the forward scan. All potentials are given with respect to the RHE. The color scale is chosen for comparison with upcoming figures that show a larger spread of SH counts. The SH image does not have any specific features and the SH signal is homogeneous with small stochastic deviations in its magnitude. The black line indicates the position of the line scan displayed in Fig. 2B. This line scan shows that the SH counts across the image range from $7.0 \cdot 10^{3}$ to $9.7 \cdot 10^{3}$ with a standard deviation of $0.5 \cdot 10^{3}$. Fig. $2 \mathrm{C}$ shows the cyclic voltammogram (black curve) together with the mean SH signal of the whole image as a function of the applied potential ( $\mathrm{SH}-\mathrm{V})$. Just before the SH imaging, the gold electrode was electrochemically cleaned by cycling through oxidizing and reducing potentials, until we obtained a stable cyclic voltammogram, as described in the Materials and Methods. The cyclic voltammogram shows the features that are expected for gold elec$\operatorname{trodes}^{56,58}$ and three different regions can be identified in the forward scan. The first region is termed the polarization or double layer charging region $(0 \text { to } 0.4 \mathrm{~V})^{59}$ with no/minor surface anion adsorption (for the weakly adsorbing ions we employ here) ${ }^{57,60}$. In this range, only a change in the electrostatic charge on the metal and corresponding change in double layer structure is observable. ${ }^{58}$ The second region $(0.4$ to $1.2 \mathrm{~V})$ is a region of further polarization where anion adsorption starts after the point of zero charge (in our case $0.4 \mathrm{~V}$, as detailed further below in the text $)^{59}$ with strong adsorption of phosphate species occurring between $\sim 0.7-1.2 \mathrm{~V}{ }^{60}$ The third region corresponds to oxide growth $(1.2$ to $1.7 \mathrm{~V}) .{ }^{61}$ It has been shown that lowcoordinated gold atoms exhibit lower activation barriers. ${ }^{62}$ Polycrystalline gold generally exhibits a large number of surface defects, and we expect that a considerable amount of these defect will be low-coordinated gold surface atoms. Therefore, the slight peak in the CV near $1.2 \mathrm{~V}$ in the forward scan is likely caused by the pre-monolayer oxidation of surface steps and defects. ${ }^{63}$ The peaks above $1.35 \mathrm{~V}$ are the result of oxidation of the first full layer of metal atoms including terraces, which happens by replacement of adsorbed anions by either $\mathrm{O}$, or $\mathrm{OH}$, and subsequent site exchange of the oxygen species with metal ions, ${ }^{64-67}$ while subsequent layers of oxide are only expected to grow at higher potentials. In the reverse scan, the peak around $1.1 \mathrm{~V}$ results from the reduction of the oxide. The small peak around $0.4 \mathrm{~V}$ is due to a minor impurity (see SI for a more detailed discussion). The slight bend in the cyclic voltammogram from 0 to $0.5 \mathrm{~V}$ is due to the presence of oxygen that causes a negative current due to the oxygen reduction reaction. ${ }^{44}$

During the forward scan, the spatially-averaged SH intensity increases and eventually reaches a maximum around $1.1 \mathrm{~V}$ (Fig. 2C). The SH intensity then decreases from 1.2 to $1.7 \mathrm{~V}$. In the reverse scan, the $\mathrm{SH}$ signal recovers to its initial highest value once the surface oxide is 
fully reduced at $1.1 \mathrm{~V}$ vs. RHE, displaying a pronounced hysteresis. Note that during forward and reverse scans an identical parabolic behavior of the SH intensity can be observed as a function of applied bias between 0 and $0.9 \mathrm{~V}$. The mean SH signal intensity of the nanocrystalline electrode as a function of applied voltage shows similar features to what has been already reported in the literature for both silver and gold electrodes ${ }^{33,40,41}$ and matches well with the CV data: from 0 to $1.2 \mathrm{~V}$ the $\mathrm{SH}$ intensity is expected to increase with increasing positive bias due to the increasing (positive) charge density at the metal surface. The subsequent decrease with increasing positive bias above $1.2 \mathrm{~V}$ is the result of gold surface oxidation. ${ }^{31,33}$

A

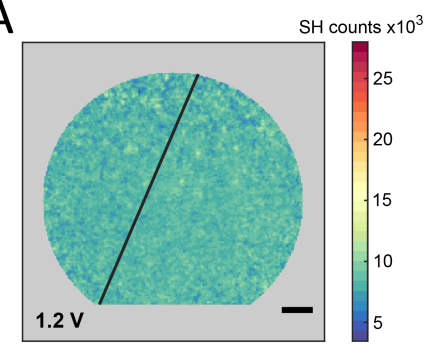

B

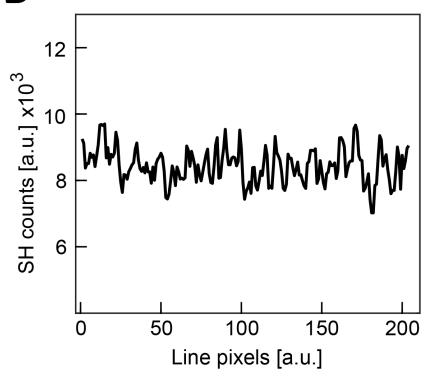

C

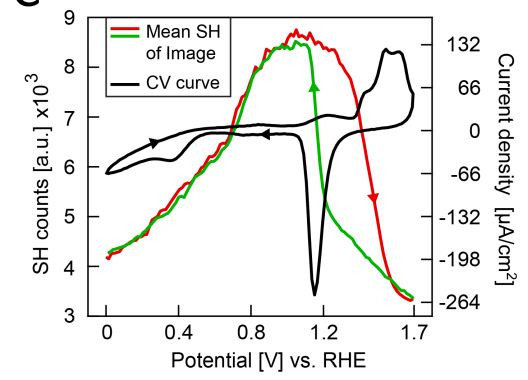

Figure 2: Second harmonic imaging of the nanocrystalline gold electrode in PPP polarization as a function of applied voltage. (A) The SH image at $1.2 \mathrm{~V}$ vs. RHE during the forward scan. The SH image is a frame extracted from a SH video recorded with $0.25 \mathrm{~s}$ acquisition time per frame. The $\mathrm{CV}$ sweeping rate was set to $60 \mathrm{mV} / \mathrm{s}$ and the electrolyte was $0.5 \mathrm{M} \mathrm{NaH}_{2} \mathrm{PO}_{4}$. (B) The variation of $\mathrm{SH}$ counts across the image was obtained from the black line scan in (A). (C) Cyclic voltammogram (black curve) displayed with the mean SH signal of the whole image. The red and green traces are recorded during the forward and reverse scan, respectively. The potential values are given with respect to the RHE. Scale bar is $10 \mu \mathrm{m}$.

Fig. 3A, B, and $\mathrm{C}$ show the $\mathrm{SH}$ images of the polycrystalline gold electrode recorded during $\mathrm{CV}$ at $60 \mathrm{mV} / \mathrm{s}$ in a phosphate $0.5 \mathrm{M} \mathrm{NaH}_{2} \mathrm{PO}_{4}$ buffer solution $(\mathrm{pH}=2.8)$ at three different potentials during the forward scan: $0.2 \mathrm{~V}, 1.2 \mathrm{~V}$ and $1.51 \mathrm{~V}$ vs. RHE. The SH images were recorded in the PPP polarization combination. The SH signal in all three images, Fig. 3A, $\mathrm{B}$, and $\mathrm{C}$, display strong spatial heterogeneity. The color scale is identical to the color scale of Fig. 2A, offering a direct comparison of the nanocrystalline vs. the polycrystalline gold electrode. The line scan in Fig. 3B shows the variation of the SH counts across the image ranging from $4.4 \cdot 10^{3}$ to $50 \cdot 10^{3}$ with a standard deviation of $\sim 7.6 \cdot 10^{3}$ and an average value of $\sim 8.4 \cdot 10^{3}$ counts. For the polycrystalline gold electrode, clear areas with SH intensity per pixel above $10^{4}$ counts spanning several tens of microns can be identified, while no such areas are visible on the nanocrystalline gold electrode, where the line scan across the image is flat and the SH intensity has an average value of $\sim 8.4 \cdot 10^{3}$ counts. The mean SH intensity of the entire field of view shown in the second panel of Fig. 3D (black curve) has a maximum around $1 \mathrm{~V}$ and is qualitatively very similar to the mean SH intensity recorded for the nanocrystalline electrode 
shown in Fig. 2C., with a maximum around 1.1 V. However, it is interesting to note that different spots on the surface show distinct SH behavior as a function of applied potential, therefore suggesting that looking only at the averaged SH signal obscures the local variations. This is demonstrated in Fig. 3D, where the SH intensity dependence on the applied potential is shown for three representative pixels, P1 (blue lines), P2 (red lines), and P3 (green lines) for three consecutive CV cycles, named CV1, CV2 and CV3. Pixels P1 and P3 are from neighboring bright (higher signal intensity) and dark (lower signal intensity) SH patches, respectively. Pixel P2 lies on their border. For further analysis, we highlight two regions of interest: the polarization region (PR), marked in yellow in Fig. 3D and the oxide growth (OG) region, marked in orange. Only the forward scan will be considered here. In the PR, the SH intensity of pixel P1 increases with applied potential, the SH intensity of pixel P3 slightly decreases with increasing potential, and the SH signal for pixel P2 increases slightly. On the other hand, in the OG region, all three pixels exhibit qualitatively similar SH behavior, i.e. the SH intensity decreases with increasing potential. Lastly, inspecting the SH signal on the whole forward scan $(0.1-1.6 \mathrm{~V})$, it appears that the borderline pixel $\mathrm{P} 2$ shows an intermediate $\mathrm{SH}$ behavior between pixels P1 and P3, suggesting that this transition region is an average between two opposite trends. The nanocrystalline sample at three different potentials during the forward scan is shown in the SI for comparison (see SI, Fig. S3). The SH intensity quadratically increases with increasing bias in the PR for the three pixels displayed.

Prior work has established that the efficiency of SH generation is highly dependent on the exposed surface structure ((111) vs. (110) facet, for example) and for a given facet it is also strongly dependent on the azimuthal orientation of the facet. ${ }^{35,36}$ This could possibly explain the difference of overall magnitudes in SH-V curves belonging to the three different pixels in Fig. 3D. However, this alone is unlikely to explain why some pixels display a decreasing SH signal with increasing bias in the PR region. An interpretation for the behavior of these pixels will be discussed in the next section. Interestingly, the voltage dependence in the OG region appears to be common to all pixels, indicating that once the oxide is starting to grow, the different contributions to the SH signal observed in the PR do not play a significant role anymore in the OG potential window. 


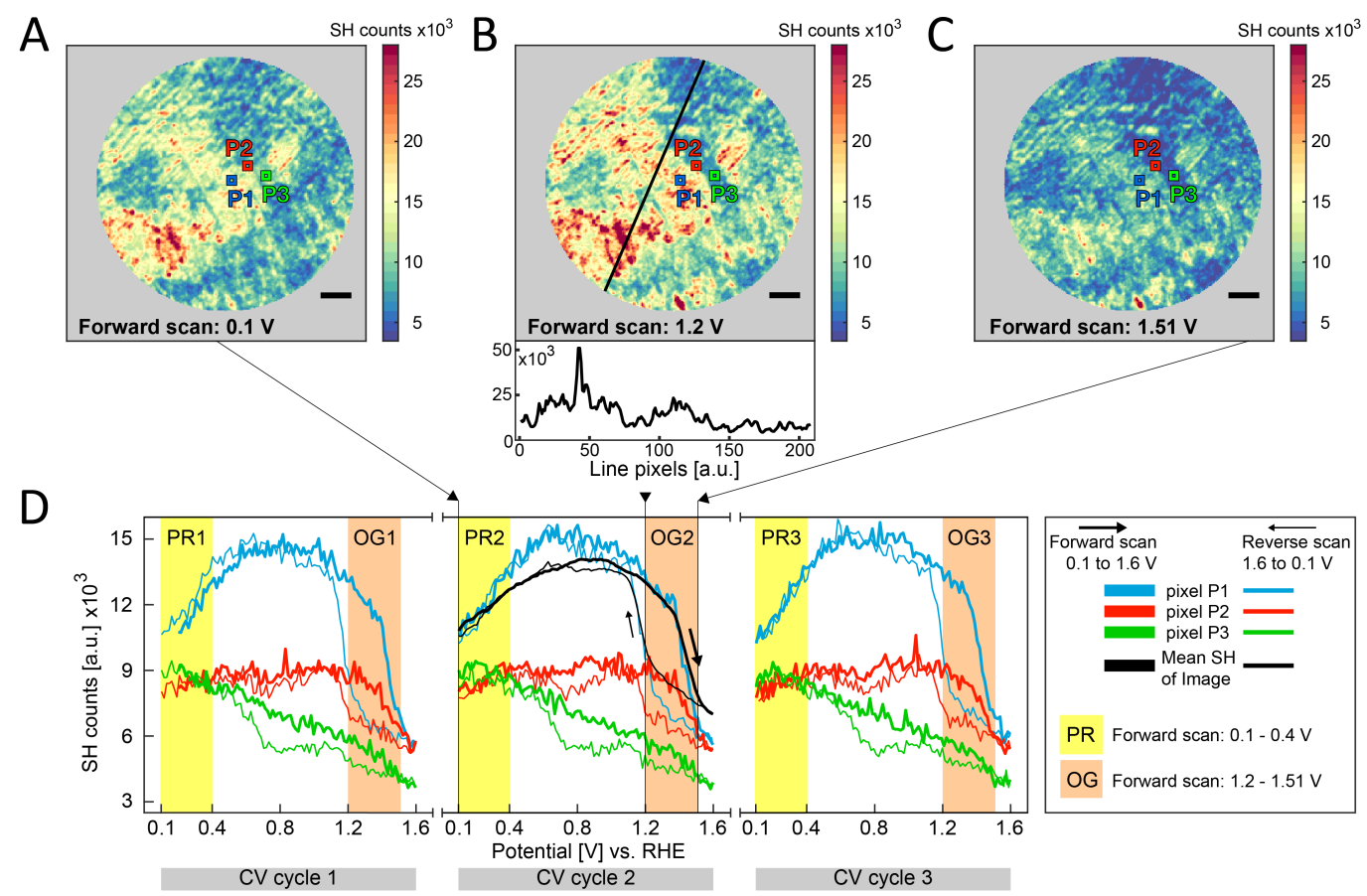

Figure 3: Second harmonic imaging of the polycrystalline gold electrode in PPP polarization as a function of applied voltage. (A), (B), (C) SH images at $0.1 \mathrm{~V}, 1.2 \mathrm{~V}$, and $1.51 \mathrm{~V}$ vs. RHE during the forward $60 \mathrm{mV} / \mathrm{s}$ scan. The SH images are frames extracted from a SH video recorded with $0.25 \mathrm{~s}$ acquisition time per frame. Points $\mathrm{P} 1$, P2, and P3 mark the chosen single pixel locations. The color scale is identical to Fig. 2A. In (B) the black line shows variation in SH counts across the image. (D) SH intensity as a function of the potential applied to the gold electrode in three continuous CV cycles. The blue curve corresponds to pixel P1; the red curve corresponds to pixel P2; and the green curve corresponds to pixel P3. The black line in the second image corresponds to the average SH signal of the whole image in $\mathrm{CV}$ cycle 2. The thick lines correspond to the forward scan, while the thin lines correspond to the reverse scan. The polarization region (PR, 0.1-0.4 V) is marked in yellow for all three cycles while the oxide growth regions $(\mathrm{OG}, 1.2-1.51 \mathrm{~V})$ is marked in orange. The potential values are given with respect to the reversible hydrogen electrode. Scale bar is $10 \mu \mathrm{m}$.

\section{Dependence of SH intensity on applied potential for polycrystalline Au}

Characterizing the SH signal and all of its contributions is a difficult task even on well-defined single crystal samples. Here, the situation is even more complicated as we use polycrystalline gold with no prior knowledge of the gold morphology at each pixel. In spite of this complication, we can still extract useful information about the surface changes as a function of potential by analyzing the SH data within the mathematical framework described above. In Fig. 4 we show a pixel-wise fitting of the SH-V data based on Eq. (3). As described by this equation, the SH intensity can display a parabolic shape with a minimum at the potential of zero charge $V_{P Z C}$ when $\chi^{(3)^{\prime}} \gg \chi^{(2)} \cdot{ }^{33}$ The reason for this SH-V behavior is related to the increase in surface charge density: Increasing the applied potential on the electrode increases the (positive) surface charge density at the metal surface. This increases the charge gradient at the interface, which results in a quadratic increase of the SH intensity with applied bias. ${ }^{17}$ The minimum of the 
parabola can be displaced from the $V_{P Z C}$ when the relative strength of $\chi^{(3)}$ decreases with respect to $\chi^{(2)}$. In this case, the signal with increasing applied potential up to $V_{P Z C}$ is due to a superposition of an increase in (positive) surface charge density (although the charge gradient is here reduced as we are below $V_{P Z C}$ ) and of the $\chi^{(2)}$ contribution. In our case, the fundamental wavelength of our laser is $1030 \mathrm{~nm}$, and the gold response is free electron-like (no interband transition) at this wavelength. We therefore expect an important $\chi^{(3)^{\prime}}$ contribution. ${ }^{33}$ However, by examining Fig. 3D, we can already also anticipate a significant amount of $\chi^{(2)}$ contribution: Considering the half-parabola in the potential range we investigate (from 0.1 to $0.4 \mathrm{~V}$ ), it can be seen that the minimum is not located at $V_{P Z C}$ but rather shifted to more negative bias (not visible in our experiment).

The SH-V signal is fitted in the PR with a parabola according to Eq. (3) at every pixel resulting in a quadratic (Q), a linear (L) and a constant (C) coefficient. Eq. (3) is applied to the PR potential interval as the approximation made in Eq. (4) is only valid in absence of strong anion adsorption. ${ }^{33}$ For the fitting procedure, the knowledge of $V_{P Z C}$ is necessary. A large range of $V_{P Z C}$ values has been reported in the literature for different gold facets. ${ }^{58,68}$ Our polycrystalline gold electrode was prepared with the same procedure as reported by Mariano et al., ${ }^{5}$ where the gold surface contained almost exclusively (111) and (110), but almost no (100) facets. We chose for the $V_{P Z C}$ a value of $0.4 \mathrm{~V}$ vs. RHE, because it appears to be the average of the values reported in the literature. The choice of this average value will be further commented here below.

Fig. 4A, B, C, and D were calculated from PR2 (see Fig. 3D) thus the quadratic and linear coefficients have been labeled as $\mathrm{Q}_{2}$ and $\mathrm{L}_{2}$. Fig. $4 \mathrm{~A}$ and $\mathrm{B}$ show respective maps of the quadratic and linear fit coefficients. These two maps look qualitatively similar; however, their scale differs. The map of linear coefficients shows much clearer large-scale features, while the map of quadratic coefficients reveals more signal variation within these features. Fig. 4C displays again the map of quadratic coefficients, however, this time with a color coding separating the $\mathrm{Q}_{2}>0$ and $\mathrm{Q}_{2} \leq 0$. The regions where $\mathrm{Q}_{2}>0$ account for $59.7 \%$ of the total surface, while the regions where $\mathrm{Q}_{2} \leq 0$ represent $40.3 \%$ of the total surface. The negative $\mathrm{Q}_{2}$ values are present both in larger patches as well as in smaller spots distributed across most of the surface. The areas where $\mathrm{Q}_{2} \leq 0$ indicate that there is no increase in the SH intensity with increasing potential, but rather a decrease (or almost a flat dependence on the voltage), implying that in these areas the increase in (positive) surface charge density caused by the increasing potential is counteracted by another effect (see for example pixels P3 and P2 in Fig. 3D). 
We hypothesize that anion adsorption at potentials below $\mathrm{V}_{\mathrm{pzc}}$ could occur in these areas, leading to charge screening at the gold surface and therefore to a decrease in SH intensity. Additionally, anion adsorption can drive or influence electro-chemical processes even for the low applied potentials of the PR ${ }^{64,65,69}$ or oxidation of metastable surface states, ${ }^{70}$ with a corresponding modification of the SH intensity. While phosphate ions, specifically dihydrogen phosphate ions $\left(\mathrm{H}_{2} \mathrm{PO}_{4}^{-}\right)$at the $\mathrm{pH}$ of our experiment, are not expected to strongly adsorb on the surface, ${ }^{57}$ they are still expected to be better sorbents than very weakly adsorbing ions, as for example $\mathrm{ClO}_{4}^{-}$ions. ${ }^{71}$ As such, phosphate adsorption in specific areas is a plausible option. It is important to note here that model we employ assumes no/minor adsorption of ions in order to fit the regions $\mathrm{Q}_{2}>0$. Deviations from the model (regions where $\mathrm{Q}_{2}<0$ ) indicate that, in those regions, the SH intensity as a function of applied potential in the PR cannot be interpreted within the framework established by Eq. (6). Therefore, the anomalous behavior of those specific areas can be tied to ion adsorption. This phenomenon could be the manifestation of a local $V_{\text {PZC }}$ being different than the average $V_{\text {PZC }}$ we have assumed for our sample. ${ }^{72}$ As the choice of the average $V_{\text {PZC }}$ was made for the purposes of the fitting, any deviations from it have to be interpreted as local variations from this average value that we arbitrarily chose, which indicate that the point of zero charge is not a homogeneous quantity across the surface but has to be treated as a spatially-dependent parameter. ${ }^{72}$

Having considered the positive and negative values of $\mathrm{Q}_{2}$, we also specifically identify the regions where $\mathrm{Q}_{2}$ is close to zero. To specifically identify pixels where $\mathrm{Q}_{2}$ is close to zero, we first need to define a threshold. Considering that the maximum $\mathrm{Q}_{2}$ values are close to $40 \cdot 10^{3}$, a threshold value of $\left|\mathrm{Q}_{2}\right| \leq 1000$ represents $2.5 \%$ of the maximum $\mathrm{Q}_{2}$ values, and all the pixels with values below this threshold can be considered as having negligible $\mathrm{Q}_{2}$. Only a small number of pixels where $\mathrm{Q}_{2}$ is close to zero $(\sim 3.8 \%$ of the total surface) is found, reflecting that there are only very few areas on the surface where the SH signal linearly depends on the voltage $\left(\chi^{(2)} \gg \chi^{(3)^{\prime}}\right)$, in agreement with the fact that we do not use a fundamental wavelength close to the interband transition regime of gold. Additionally, we find an even smaller number of pixels where $\mathrm{Q}_{2}$ and $\mathrm{L}_{2}$ are both close to zero $\left(\sim 2.4 \%\right.$ of the total surface when $\left|\mathrm{Q}_{2}\right|$ and $\left|\mathrm{L}_{2}\right| \leq$ 1000 ), where the SH signal remains insensitive to the applied voltage.

For comparison, the SH-V dependence of the nanocrystalline sample mostly shows regions where the SH intensity quadratically increases with increasing bias in the PR, with only a small number of pixels with an inverted potential-dependence scattered across the image (see SI, Fig. S4).

Having determined the surface areas where the SH intensity quadratically increases with the applied potential and the ones where this is not the case, we can now correlate these voltage-dependent behaviors with specific surface characteristics. In Fig. 4D we examine the results of fitting the data to Eq. (3) as the ratio of the $\mathrm{Q}_{2}$ map over the absolute value of the $\mathrm{L}_{2}$ 
map. The absolute value $\mathrm{L}_{2}$ is chosen here to focus on the $\mathrm{Q}_{2} / \mathrm{L}_{2}$ ratio without distinctions between $\pm \mathrm{L}_{2}$ (the sign of $\mathrm{L}_{2}$ is related to the sign of the surface susceptibility, which for simplicity we do not consider in this analysis). By inspecting the ratio $\mathrm{Q}_{2} /\left|\mathrm{L}_{2}\right|$ of different pixels, we can compare the relative strength of $\chi^{(3)^{\prime}}$ over $\chi^{(2)}$ between these pixels according to Eq. (6). Only pixels with $\mathrm{Q}_{2}>0$ are considered here, as pixels with $\mathrm{Q}_{2}<0$ do not satisfy the assumptions of Eq. (6). As a reference value, the magnitude of $\chi^{(2)}$ for gold at $1064 \mathrm{~nm}$ was reported to be close to $2 \cdot 10^{-19} \mathrm{~m} \cdot \mathrm{V}^{-1},{ }^{51}$ which is close to the magnitudes reported for $\chi^{(3)}$ for gold, in the range of $2 \cdot 10^{-19}$ to $7 \cdot 10^{-19} \mathrm{~m} \cdot \mathrm{V}^{-1}$ at $1030 \mathrm{~nm} ;{ }^{54} \chi^{(3)}$ is not equal to $\chi^{(3)}$ but is considered to be in the same order of magnitude for a metal. ${ }^{33}$ The ratio $\mathrm{Q} /|\mathrm{L}|$ is then expected to be in the range of $0-10$, which agrees with our data showing the majority of the pixels displaying a ratio between 0 and 4 . Values below 1 (20.65\% of the total surface) are colored in blue and values above 1 (79.35\% of the total surface) are colored in yellow to red. A detailed breakdown of the percentage of pixels per location is presented in Table 1. These percentages show that pixels with higher second-order surface susceptibility $\left(\mathrm{Q}_{2} /\left|\mathrm{L}_{2}\right|<1\right)$ tend to accumulate at the edges of distinct surface features and at the borderline with the greyed areas $\left( \pm \mathrm{Q}_{2}\right.$ boundary). On the other hand, the areas where $\mathrm{Q}_{2} /\left|\mathrm{L}_{2}\right|>1$ are more evenly distributed over the image and there is not as much specific accumulation at the $\pm \mathrm{Q}_{2}$ boundary.

Table 1: Percentage of pixels in Fig. 4D according to the fitting the SH data of polycrystalline gold to Eq. (3)

\begin{tabular}{|c|c|c|c|c|c|}
\hline \multicolumn{3}{|c|}{$\mathrm{Q}_{2}>0, \mathrm{Q}_{2} /\left|\mathrm{L}_{2}\right|>1$} & \multicolumn{3}{c|}{$\mathrm{Q}_{2}>0, \mathrm{Q}_{2} /\left|\mathrm{L}_{2}\right|<1$} \\
\hline Total & $\pm \mathrm{Q}_{2}$ boundary & elsewhere & Total & $\pm \mathrm{Q}_{2}$ boundary & elsewhere \\
\hline $79.35 \%$ & $40.68 \%$ & $38.67 \%$ & $20.65 \%$ & $14.21 \%$ & $6.44 \%$ \\
\hline
\end{tabular}

From these results we see that, in the areas with $\mathrm{Q}_{2}>0$, the majority of the pixels $(79.35 \%)$ display a relatively stronger $\chi^{(3)^{\prime}}$ contribution, indicating that the $\mathrm{SH}$ signal is dominated by variations of surface charge density. A minority of pixels in the $\mathrm{Q}_{2}>0$ areas display a stronger $\chi^{(2)}$ contribution (20.65\%), which indicates that the signal is dominated by variations of the surface term ${ }^{33}$ and it is likely accompanied by the excitation of a local surface plasmon (LSP). While surface plasmons in general cannot be excited by transverse electromagnetic waves by a simple one-beam reflection on a perfectly flat gold surface, the presence of irregularities at the surface can facilitate the excitation of the LSP. ${ }^{73,74}$ Thus, we can assign the areas with the highest $\chi^{(2)}$ contribution $\left(\mathrm{Q}_{2} /\left|\mathrm{L}_{2}\right|<1\right)$ to various defects, grain boundaries, or small roughened parts that are spread over the polycrystalline surface. This is further supported by the observation of the majority of the pixels with stronger $\chi^{(2)}$ contribution accumulating 
at the edges between surface features displaying different $\mathrm{SH}$ intensity, i.e. grain boundaries ( $14.21 \%$ out of $20.65 \%$ total pixels).

Regions where $\mathrm{Q}_{2}<0$ (SH intensity decreases with potential) cannot be interpreted within the framework established by Eq. (6), as we hypothesized that the initial assumption of no (strong) anion adsorption is not met in those specific areas. We can further speculate about the nature of these areas by inspecting Fig. 4E. Fig. 4E shows the $\mathrm{Q}_{3} / \mathrm{L}_{3}-\mathrm{Q}_{2} / \mathrm{L}_{2}$ difference maps for PR3 and PR2 with no distinctions between $\mathrm{Q}>0$ or $\mathrm{Q} \leq 0$. Here, a higher difference, either positive or negative, indicates that the shape of the SH-V curve has significantly changed between CV2 and CV3 cycle. This map evidences that the areas with the largest heterogeneity and the largest changes cycle to cycle are the borders of $+Q /-Q$ areas. As a quantitative example, we estimate the percentage of pixels with values $>2$ or $<-2$ to be $40 \%$, which correlates well with the percentage of pixels where $\mathrm{Q}_{2}<0$ (40.3\%), as determined above. A detailed breakdown of the percentage of pixels per location is presented in Table 2 and highlights that the borderline regions are prone to change cycle to cycle, which is a similar observation as in Fig. 4D. On the other hand, the areas in Fig. 4E that change the less cycle to cycle match well with the regions where $\mathrm{Q}_{2}>0$ (yellow areas in Fig. $4 \mathrm{C}$ ).

Table 2: Percentage of pixels in Fig. 4E according to the fitting the SH data of polycrystalline gold to Eq. (3)

\begin{tabular}{|c|c|c|}
\hline \multicolumn{3}{|c|}{$\mathrm{Q}_{3} / \mathrm{L}_{3}-\mathrm{Q}_{2} / \mathrm{L}_{2}>2$ or $<-2$} \\
\hline Total & $\pm \mathrm{Q}_{2}$ boundary & elsewhere \\
\hline $40 \%$ & $27.2 \%$ & $12.8 \%$ \\
\hline
\end{tabular}

The observation of large differences from cycle to cycle, together with a higher heterogeneity, in most of the areas where the quadratic coefficient is negative suggests that these surface areas undergo dramatic structural changes while potential cycling. These findings support our hypothesis hereabove, where anion adsorption behavior below the average VPZC could be the cause for increased surface reconstruction. Indeed, surface reconstruction induced by specific adsorption of ions has been previously observed by SHG on gold samples. ${ }^{34,35}$ LSP excitation, related to the second order susceptibility, is also possible in these regions, but is difficult to quantify in this case using the linear coefficient $\mathrm{L}$, which in practice indicates the position of the minimum of the parabola. As a matter of fact, the position of this minimum could also be shifted as a result of anion adsorption ${ }^{41}$ and not only due to LSP excitation. 


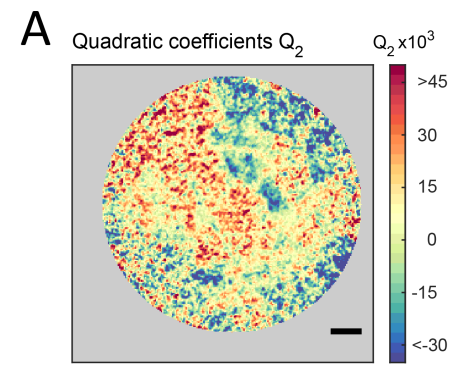

D
B

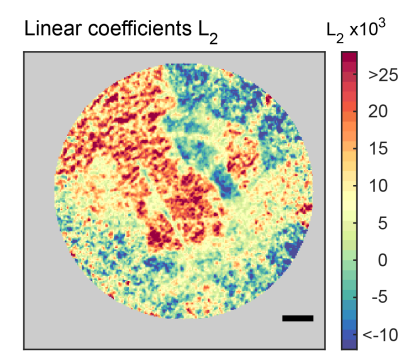

$\mathrm{E}$

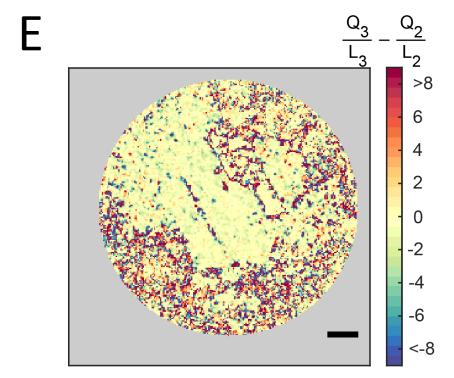

C

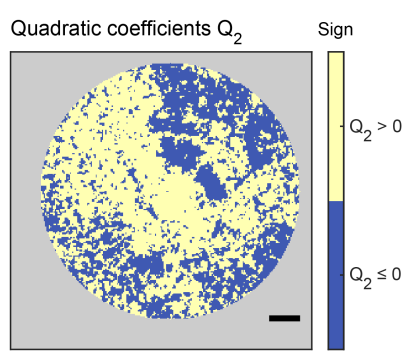

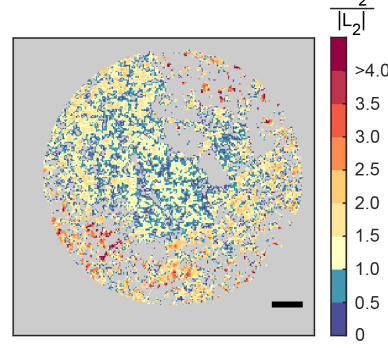

Figure 4: Fitting of the second harmonic signal in the PR as a function of applied potential for polycrystalline gold electrode. Second harmonic PPP polarization signal corresponding to potential interval PR2 (see Fig 3D) is fitted by a polynomial of $2^{\text {nd }}$ degree with point of zero charge offset common to all pixels. This yields a map of fitted quadratic coefficients $\mathrm{Q}_{2}$ in (A) and a map of fitted linear coefficients $\mathrm{L}_{2}$ in (B). (C) Map of quadratic coefficients $\mathrm{Q}_{2}$ with a binary color-code of yellow $\left(\mathrm{Q}_{2}>0\right)$ and blue $\left(\mathrm{Q}_{2} \leq 0\right)$. (D) Division of $\mathrm{Q}_{2}$ map over the absolute value of $\mathrm{L}_{2}$ map. Pixels where $\mathrm{Q}_{2} \leq 0$ are not considered here. (E) Difference of quadratic over linear coefficients ratios obtained from potential interval PR3 minus PR2 (see Fig 3D). Scale bar is $10 \mu \mathrm{m}$.

\section{Correlation coefficient analysis}

The analysis of the data with Eq. (3) is limited to the PR and Q $>0$ regions where Eq. (4) holds. We now describe an alternative image processing method in order to support our findings from above and that can be applied to other potential intervals than the PR. The aim of this method is to distinguish the differences in shapes of two SH-V signals in the same potential interval, however, from two consecutive CV cycles. Such a method can identify which pixels show different $\mathrm{SH}-\mathrm{V}$ behavior in one $\mathrm{CV}$ cycle compared to the previous $\mathrm{CV}$ cycle, pointing to potential-dependent surface changes. For this reason, we chose to calculate the correlation coefficient for every pixel as follows:

$$
\operatorname{Corrcoeff}(A, B)_{\text {per pixel }}=\frac{\operatorname{cov}(A, B)}{\sigma_{A} \sigma_{B}},
$$

where $A$ is the respective $\mathrm{SH}-\mathrm{V}$ signal intensity at a given pixel, and $B$ is the mean SH-V signal intensity of the whole image, both for a given potential interval. $\operatorname{cov}(A, B)$ is the covariance of $A$ and $B, \sigma_{A}$ is the standard deviation of $A$ and $\sigma_{B}$ is the standard deviation of $B$. The correlation coefficient is a measure for the correlation of $A$ and $B$ as a function of voltage. Its output value ranges from -1 to 1 . A value of 1 expresses an ideal correlation between $A$ and $B$ 
: for a positive increase in $A-$ there is also a positive increase in $B$. The other extreme of -1 expresses an ideal inverse correlation between $A$ and $B$ : for a positive increase in $A$ - there is a decrease in $B$. A value of 0 implies that there is no correlation between $A$ and $B$.

Using Eq. (7), we calculated the correlation coefficient for every pixel using SH-V curves from $3 \mathrm{CV}$ cycles. The results are displayed in Fig. 5A. The red to orange areas display the strongest correlation, thus indicative of the regions where the SH-V data at each pixel behaves similarly to the averaged SH-V signal. Note that there are similarities between the red regions in Fig. 5A and the areas displayed in yellow on Fig. 4C (i.e. areas where $\mathrm{Q}_{2}>0$ ). On the other hand, the yellow to blue areas indicate regions where the $\mathrm{SH}-\mathrm{V}$ data deviates from the averaged SH-V signal. Those areas match well with blue regions in Fig. $4 \mathrm{C}\left(\mathrm{Q}_{2}<0\right.$ areas $)$.
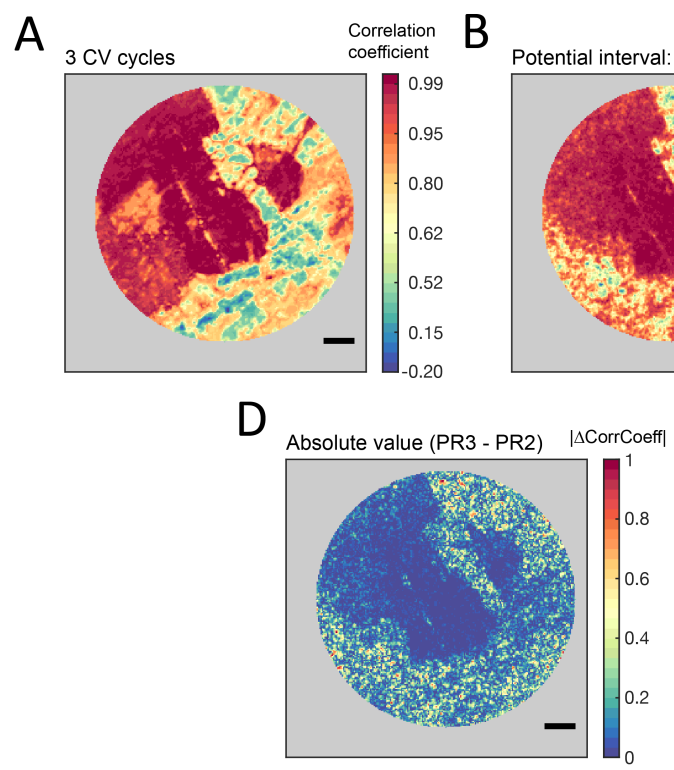

B

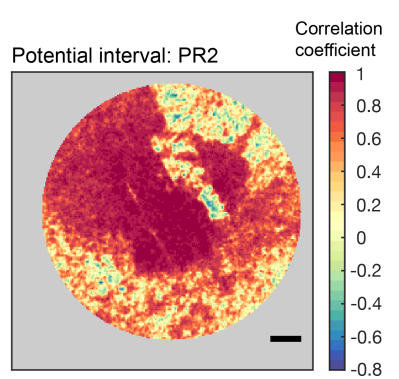

$\mathrm{E}$

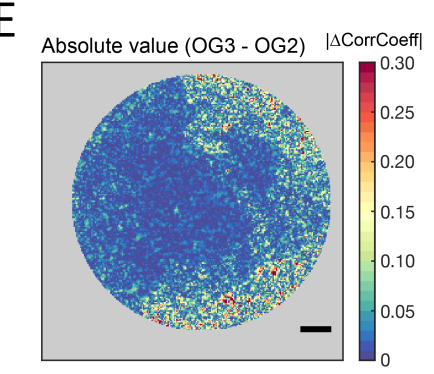

Figure 5: Correlation coefficient analysis. (A) Correlation coefficient map of the polycrystalline gold electrode calculated from three full CV cycles. (B), (C): Correlation coefficient maps calculated from PR2 and PR3 potential intervals, respectively. (D) Absolute value of the difference between (B) and (C). (E) Absolute value of the difference between two correlation maps calculated in potential intervals OG3 and OG2. SH data was recorded in the PPP polarization combination. Scale bar is $10 \mu \mathrm{m}$.

We obtain additional information by performing the correlation coefficient analysis also on the PR and OG potential intervals (see Fig. 3D). As mentioned above, the first potential interval $(0.1-0.4 \mathrm{~V})$ was chosen as the potential interval corresponding to double layer charging, where on average no/minor ion adsorption is expected. The OG region spans from 1.2 to $1.51 \mathrm{~V}$ for the polycrystalline sample. In this potential interval, the first layer of oxide is growing and eventually fully covering the surface ${ }^{64,67}$ Correlation coefficient maps are calculated for both PR and OG potential intervals in the second CV cycle (PR2, OG2) and third CV cycle (PR3, OG3). By looking at the map of the absolute difference of correlation coefficient (designated here as $\mid \Delta$ CorrCoeff $\mid$ ), we can locate surface areas where the SH-V behavior is the same, 
or different, from one cycle to the other. The correlation coefficient maps are calculated for a specific interval; however, their difference retains the full history of the sample. For example, a large value of $\mid \Delta$ CorrCoeff $\mid$ (PR3-PR2) indicates that changes have occurred between PR3 and PR2, however these changes could have originated at any point in the cycle and not only in PR2 or PR3 (also during OG2 or reduction, for example). Analogously, a large value of $\mid \Delta$ CorrCoeff $\mid$ (OG3-OG2) indicates that changes have occurred between the OG3 and OG2 region, but those changes could have originated either during the oxide growth (OG2 or OG3), or during the successive reduction and polarization of the sample in PR3. ${ }^{a}$ Note that here we consider the difference, so both correlation coefficients have to be calculated with respect to the same reference curve $B$ in Eq. (7). Here, we chose a simple $y=x$ curve as reference. ${ }^{\mathrm{b}}$ We applied this approach to the polycrystalline gold sample and present the results in Fig 5B-F. Fig. 5B,C show the calculated correlation maps from PR2 and PR3 potential intervals, which are qualitatively similar to the map calculated with respect to the average SH-V signal in Fig. 5A. Fig. 5D then shows the absolute value of the difference between Fig. 5B and C.

The $\mid \Delta$ CorrCoeff $\mid$ map shown in Fig. 5D shows large spatial heterogeneity over the surface. As mentioned above, the areas with largest values of $\mid \Delta$ CorrCoeff $\mid$ indicate that more changes are occurring in those areas over one full cycle. We take $\mid \Delta$ CorrCoeff $\mid>0.1$ as an indication for substantial changes occurring to the surface (the error on $\mid \Delta$ CorrCoeff $\mid$ is estimated to $+/-0.04$, see SI section S8). Fig. 5E also shows spatial heterogeneity in $\mid \Delta$ CorrCoeff $\mid$ calculated for the OG region. While the magnitude of the $\mid \Delta$ CorrCoeff $\mid$ cannot be directly compared for the PR and OG region, ${ }^{\mathrm{c}}$ we can compare the spatial extent of the changes on both potential intervals. In the OG region, values of $\mid \Delta$ CorrCoeff $\mid>0.05$ (the error on $\mid \Delta$ CorrCoeff $\mid$ is estimated to $+/-0.02$, see SI section S8), indicating areas with changes occurring over one full cycle, are found with a similar spatial distribution as Fig. 5D. The percentage of the total surface displaying changes of $\mid \Delta$ CorrCoeff $\mid>0.1$ in the PR interval is $39.7 \%$. For comparison, the percentage of the total surface displaying changes of $\mid \Delta$ CorrCoeff $\mid>0.05$ in the OG interval is $22.7 \%$. The nature of the changes associated with larger $\mid \Delta$ CorrCoeff $\mid$ values will be addressed below. Results for the polycrystalline sample can be compared with the nanocrystalline gold sample shown in the SI (Fig. S5). This comparison highlights that no specific

\footnotetext{
${ }^{a}$ The correlation coefficient analysis can only be used to compare the relative shape of the SH-V curves; it cannot be used directly to examine if a certain process started at a lower or higher potential.

${ }^{b}$ This ensures that the SH-V signal will be correlated against the same reference curve, therefore, exposing mutual differences. The mean SH-V signal of the whole image is not appropriate here because it can vary cycle to cycle.

${ }^{c}$ While the $\mid \Delta$ CorrCoeff $\mid$ can be calculated for each of these regions, comparing their magnitudes is challenging as the possible contributions to the SH signal are likely potential dependent.
} 
surface feature can be observed in the nanocrystalline electrode within our submicron resolution, while for the polycrystalline sample we can clearly distinguish areas whose SH-V profiles change cycle to cycle and areas that do not.

Given that the potential interval PR in Fig. 5D corresponds to the same potential interval considered in Fig. 4, we now can compare the correlation coefficient analysis in the PR with the analysis based on Eq. (3) presented above. The correlation coefficient analysis reveals that changes in the SH-V shape from one cycle to the next (regions with higher value of $\mid \Delta \mathrm{CorrCo}-$ eff $\mid$ ) occur in the same spatial regions where the analysis based on Eq. (3) (the result of which are shown in Fig. 4) yields negative quadratic coefficients. There is indeed a remarkable agreement between the percentage of total surface displaying changes of $\mid \Delta$ CorrCoeff $\mid>0.1$ in the PR interval (39.7\%) and the percentage of total surface where $\mathrm{Q}<0$ (40.3\%) obtained from the analysis of Fig. 4. At the same time, the areas with the largest $\mathrm{Q}_{3} / \mathrm{L}_{3}-\mathrm{Q}_{2} / \mathrm{L}_{2}$ values shown in Fig. 4E, indicating the largest changes cycle to cycle, also match well with the areas displaying the largest $\mid \Delta$ CorrCoeff $\mid$ values in Fig. 5D. It is therefore reasonable to assume that the correlation coefficient analysis can be used to further investigate the areas where $\mathrm{Q}<0$, a prospect that the analysis with Eq. (3) does not offer. Areas displaying a higher value of $\mid \Delta$ CorrCoeff $\mid$ (i.e. $\mid \Delta$ CorrCoeff $\mid>0.1$ ) imply a change in the surface structure/chemistry during the electrochemical cycling, which alters the SH-V behavior from one cycle to the next. On the other hand, the areas with lower values of $\mid \Delta$ CorrCoeff $\mid$ indicate that the SH-V behavior in a given potential interval is similar after one cycle. We do not expect electrochemical reactions to happen in the potential interval of $0.1-0.4 \mathrm{~V}$ and therefore assume that the largest values of $\mid \Delta$ CorrCoeff $\mid$ (PR3-PR2) in Fig. 5D originate from surface restructuring during one entire potential cycle, likely roughening the surface by oxidation and reduction. This further supports the hypothesis formulated within the SH-V analysis of Fig. 4E, where the regions with $\mathrm{Q}_{2}<0$ correspond to the areas with the largest surface changes during electrochemical cycling.

Note also that the observation of similar areas of considerable correlation differences ( $\mid \Delta$ CorrCoeff $\mid>0.05$ ) in the OG potential interval (Fig. 5E) indicates that the SH-V behavior during oxide growth does experience changes from one cycle to the next. We speculate that the $\mathrm{SH}-\mathrm{V}$ behavior during oxide growth is also affected by surface topographical changes due to potential-induced surface reconstruction. These surface topographical changes may already happen before the OG region and are possibly a consequence of surface reconstruction ${ }^{64,65,69}$ or oxidation of metastable surface states, ${ }^{70}$ processes that are very likely strongly influenced by anion adsorption. These processes could also explain areas with elevated $\mid \Delta$ CorrCoeff $\mid$ in the OG region. Because anion desorption precedes oxide growth (as detailed above), we assume that a different local potential is required to initiate oxide growth in areas where anions behave different from the average (e.g. in $\mathrm{Q}<0$ areas). Such a behavior would also affect the SH-V curve and modify the distribution of $\mid \Delta$ CorrCoeff $\mid$ values in the OG region. On the other hand, 
the rest of the areas show an identical SH-V behavior in the oxide growth region from one cycle to the next.

The difference of the correlation coefficient analysis over the analysis based on Eq. (3) is that, as far as the electrochemical behavior of the sample of interest is known, the correlation coefficient analysis can be performed in any potential interval of interest and is not limited to the PR.
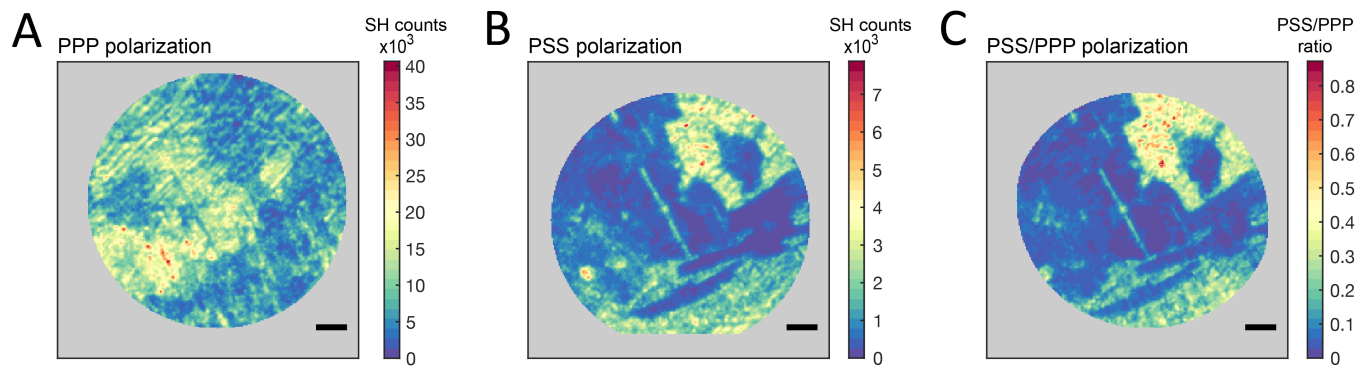

Figure 6: Second harmonic images of different polarization combinations. All three images represent the mean image averaged from the video frames corresponding to PR2 potential interval. (A), (B), and (C) correspond to PPP, PSS, and PSS divided by PPP polarization combinations for the polycrystalline gold electrode, respectively. Scale bar is $10 \mu \mathrm{m}$.

\section{PSS/PPP polarization combination ratio}

Here we present a way of identifying regions more prone to surface reconstruction during potential cycling, which possibly have a higher catalytic activity, that does not require a lengthy image analysis but relies purely on a measurement. The SH intensity from a given polarization combination is composed of multiple contributions, that unfortunately we cannot separate in our SH data. However, different facets on the surface can have a different response as a function of the incoming beam polarization. Fig. 6 shows two different polarization combinations: PPP, PSS, and their ratio for the polycrystalline gold electrode. It can be observed that these two polarization combinations appear to be complementary: the regions of highest SH intensity in the PPP polarization combination are also the regions of lowest SH intensity in the PSS polarization combination, and vice-versa. Furthermore, the areas with the strongest SH intensity signal in PPP polarization combination in Fig. 6A bear some spatial similarities to the blue areas in Fig. 5D with the lowest correlation difference between CV cycle 2 and 3, while the areas with the strongest SH intensity signal in PSS polarization in Fig. 6B display some spatial similarities with the areas of highest correlation difference in Fig. 5D. Division of PSS by PPP polarization shown in Fig. 6C presents the relative strength of the SH signal in PSS polarization in comparison with PPP polarization and visually intensifies various features at the surface. The cropping of Fig. $6 \mathrm{C}$ is a consequence of unintentionally shifted illumination when switching the polarization combinations. Here, we see that this ratio displays almost identical features as the PSS polarization combination in Fig. 6B. Indeed, the regions with a higher PSS contribution 
(given as PSS/PPP $>0.1$ ) visually correlate with the regions of higher difference in correlation coefficient observed in Fig. 5D. We estimate that $39.4 \%$ of the surface has a PSS/PPP ratio above or equal to 0.1. Comparing this number to the ones found above for the pixels with negative quadratic coefficient (40.3\%) and the pixels showing the largest correlation difference above 2 or below $-2(40 \%)$, we conclude that all three methods to visualize the data identify similar surface areas (for visual comparison of the similarity between the three methods see video in SI). No specific features can be seen on the nanocrystalline sample presented in the SI (Fig. S6) in both polarization combinations.

We note that the SH image of the PSS polarization combination in Fig. 6B is not directly superimposable with the results of the model described by Eqs. 3, 6 and the correlation analysis (obtained on images recorded in the PPP polarization combination). Some of the most apparent mismatches are the sharp diagonal thin lines in the PSS polarization combination, which we presume are due to surface scratches. The SH intensity of PPP and PSS polarization combinations are dependent on amplitudes of the isotropic, onefold, twofold and threefold symmetry contributions. ${ }^{35}$ The difference is, these amplitudes are different for these polarization combinations. Therefore, the SH intensity is dependent on the azimuthal angle of laser illumination. ${ }^{24,29}$ On top of that the SH intensity is dependent on many factors, such as i) the given polycrystalline gold surface facet and its orientation in a given pixel ${ }^{36}$ ii) occurrence of surface reconstruction; ${ }^{34,35}$ iii) anion adsorption and desorption; ${ }^{36}$ iv) LSP excitation, ${ }^{74}$ etc. Considering all these factors that contribute differently to the SH-V shape and absolute $\mathrm{SH}$ signal intensity, it is interesting to see that the images in the PSS polarization combination show a fair amount of similarities to the results of the parabolic model and the correlation analysis. While the model based on Eq. (3) and the correlation analysis were comparing two different CV cycles in PPP polarization combination, the SH image of PSS polarization shown in Fig. 6B is from a single CV cycle. This suggests that the SH PSS polarization combination on this particular electrode/electrolyte system is inherently more sensitive to the areas with irreversible surface chemistry in $\mathrm{CV}$ cycling on $\mathrm{Au}$ electrodes than the PPP polarization combination. Further work would be necessary to establish if there is a correlation between a high contrast mechanism in a specific polarization combination and areas prone to potential-induced reconstruction also for other metal/electrolyte systems. Such a general correlation could then be used as a basis for large-scale material screening with the goal to locate parts of the surface that are more prone to potential-induced surface reconstruction. This is of interest for studying corrosion and potentially for studying catalytic activity, as rough and dynamic surfaces are often catalytically active. 


\section{Conclusion}

In summary, optical second harmonic ( $\mathrm{SH}$ ) imaging in a wide-field configuration combined with in-situ cyclic voltammetry is used here to investigate surface reconstruction during potential cycling of gold nanocrystalline and polycrystalline electrodes. Our findings show that, while the average SH intensity as a function of applied bias follows a similar trend as reported in prior studies, it varies drastically on the (sub)micron level for polycrystalline electrodes: Spatial averaging obscures qualitative differences in the SH response. In contrast images of nanocrystalline electrodes show no such heterogeneity. Fitting the SH signal as a function of potential in the polarization region $(0-0.4 \mathrm{~V}$ vs. RHE), where no electrochemical reactions occur and no/minor specific ion adsorption is expected, reveals two distinct dependences on the applied potential. On the majority of the surface $(\sim 60 \%)$, the SH intensity is found to quadratically increase with increasing bias $(\mathrm{Q}>0)$. This is in line with results of previous SH spectroscopy studies, where only the average SH response of the surface was probed. On the rest of the surface $(\sim 40 \%)$ the SH intensity quadratically decreases with increasing bias $(\mathrm{Q}<0)$, which has not been reported before. In areas where $\mathrm{Q}>0$, we can quantify on a pixel-wise level the extent of the contributions from the second-and third-order susceptibilities. This analysis reveals that regions with higher second-order susceptibility, which possibly have a higher localized surface plasmon contribution, tend to be located at the boundaries between areas with $(\mathrm{Q}>0)$ and $(\mathrm{Q}<0)$. Furthermore, these boundary pixels display also the highest changes in the ratio of third-order susceptibility over second-order susceptibility between successive potential cycles, showing that surface reconstruction must occur here during potential cycling. On the other hand, areas with higher third-order susceptibility contribution appear to display minimal changes from one cycle to the next and here the SH signal as a function of applied bias can be explained mainly by reversible changes in surface charging. For the areas where the SH intensity quadratically decreases with increasing bias, we attribute this inverse behavior to anion adsorption at potentials below the average potential of zero charge, which is possibly facilitated by a rough and non-equilibrated surface structure, and therefore particularly susceptible to surface reconstruction during potential cycling. Interestingly, no such areas are identified on the nanocrystalline sample within our submicron resolution.

This hypothesis is corroborated by a correlation coefficient analysis. The correlation coefficient analysis reveals that the strongest changes in the SH-V shape from one cycle to the next (regions with higher value of $\mid \Delta$ CorrCoeff $\mid$ ) occur in the same spatial regions where the fit of the SH dependence as a function of potential yields $\mathrm{Q}<0$. We find that the percentage of the total surface area that is undergoing substantial changes cycle to cycle, where $\mid \Delta$ CorrCoeff $\mid$ $>0.1$ in the PR interval, is in good agreement with the percentage of total surface where $\mathrm{Q}<0$ (39.7\% and $40.3 \%$, respectively). At the same time, the areas with the largest $\mathrm{Q}_{3} / \mathrm{L}_{3}-\mathrm{Q}_{2} / \mathrm{L}_{2}$ values, indicating the largest changes cycle to cycle, also match well with the areas displaying 
the largest $\mid \Delta$ CorrCoeff $\mid$ values. We therefore assign these largest values of $\mid \Delta$ CorrCoeff $\mid$ to originate from structural modifications occurring over one entire electrochemical cycle, likely roughening the surface. A similar spatial distribution of the highest $\mid \Delta$ CorrCoeff $\mid$ values is also obtained for the correlation coefficient analysis in a potential region where surface oxide is expected to grow, adding further evidence that $\mathrm{Q}<0$ regions are particularly prone to surface reconstruction during potential cycling. Comparison to the nanocrystalline sample highlights that no specific spatial area of increased surface reconstruction can be observed in the nanocrystalline electrode within our submicron resolution. This finding suggests that the extent of areas with a rough and non-equilibrated surface is dependent on the grain size.

Lastly, the areas with the largest SH intensity in the PSS polarization combination match reasonably well with the above identified areas that are particularly prone to surface reconstruction during potential cycling. Following this observation, we will further investigate $\mathrm{SH}$ wide-field imaging in various polarization combinations on other metal/electrolyte systems. Establishing a correlation between a high contrast mechanism in a specific polarization combination and areas prone to potential-induced surface reconstruction would be a valuable tool for the investigation of certain aspects of surface chemistry, like corrosion, or potentially catalytic activity on a polycrystalline sample without any a priori knowledge of its surface structure.

This study demonstrates the applicability of a new imaging modality to the operando characterization of electrocatalysts on hundreds of $\mu \mathrm{m}$ length scales. Due to its speed and submicron resolution, this technique could be utilized to correlate various parameters of the surface (for example it could be potentially used to map out the local $\mathrm{V}_{\mathrm{PZC}}$ ) and chemical reactivity in an application-oriented context.

\section{Supporting Information}

Illustration of one-beam illumination of the sample, the effect of surface contamination on the $\mathrm{CV}$, details on $\mathrm{CV}$ reduction peak at $0.4 \mathrm{~V}$ for the nanocrystalline gold electrode, $\mathrm{SH}$ mapping of nanocrystalline gold during $\mathrm{CV}$, analysis of $\mathrm{SH}$ potential-dependent signal for nanocrystalline gold, correlation-coefficient analysis of nanocrystalline gold, SH images of PSS and PPP polarization combinations for the nanocrystalline gold, third-order susceptibility of gold at the fundamental wavelength used in our experiments, details on processing of the $\mathrm{SH}$ videos, determining the error bars of SH images for PPP polarization combination, determining the error bars for the correlation coefficient analysis 


\section{Acknowledgements}

This work was supported by the Julia Jacobi Foundation, the Marie Skłodowska-Curie grant agreement No 721766 (S.R. and I.N.). A.M. thanks the Swiss National Science Foundation for funding through the Ambizione Grant PZ00P2_174146. R.K.C. thanks the Deutsche Forschungsgemeinschaft (DFG, German Research Foundation) for funding through the Germany's Excellence Strategy - EXC-2033 - Project Number 390677874 and the European Research Council (ERC) under the European Union's Horizon 2020 research and innovation program with grant agreement no 772286. The authors thank Wiebke Frandsen (Inorganic Chemistry department, Fritz Haber Institute) and Felix Gerke (Free University of Berlin) for SEM images, Gregor Koch (Inorganic Chemistry department, Fritz Haber Institute) for annealing of the polycrystalline gold foils and Sabine Wasle (Physical Chemistry department, Fritz Haber Institute) for the physical vapor deposited samples.

\section{References}

(1) Catalysis by Materials with Well-Defined Structures, 1st ed.; Wu, Z., Overbury, S. H., Eds.; Elsevier, 2015.

(2) Ertl, G. Reactions at Surfaces: From Atoms to Complexity (Nobel Lecture). Angew. Chemie Int. Ed. 2008, 47, 3524-3535.

(3) Somorjai, G. A. Introduction to Surface Chemistry and Catalysis; Wiley, 1994.

(4) Warren, S. C.; Voïtchovsky, K.; Dotan, H.; Leroy, C. M.; Cornuz, M.; Stellacci, F.; Hébert, C.; Rothschild, A.; Grätzel, M. Identifying Champion Nanostructures for Solar Water-Splitting. Nat. Mater. 2013, 12, 842-849.

(5) Mariano, R. G.; McKelvey, K.; White, H. S.; Kanan, M. W. Selective Increase in $\mathrm{CO}_{2}$ Electroreduction Activity at Grain-Boundary Surface Terminations. Science (80-. ). 2017, 358, 1187-1192.

(6) Mavrikakis, M.; Hammer, B.; Nørskov, J. K. Effect of Strain on the Reactivity of Metal Surfaces. Phys. Rev. Lett. 1998, 81, 2819-2822.

(7) Strasser, P.; Koh, S.; Anniyev, T.; Greeley, J.; More, K.; Yu, C.; Liu, Z.; Kaya, S.; Nordlund, D.; Ogasawara, H.; et al. Lattice-Strain Control of the Activity in Dealloyed Core-Shell Fuel Cell Catalysts. Nat. Chem. 2010, 2, 454-460.

(8) Engstfeld, A. K.; Brimaud, S.; Behm, R. J. Potential-Induced Surface Restructuring-The Need for Structural Characterization in Electrocatalysis Research. Angew. Chemie Int. Ed. 2014, 53, 12936-12940.

(9) Engstfeld, A. K.; Klein, J.; Brimaud, S.; Behm, R. J. Electrochemical Stability and 
Restructuring and Its Impact on the Electro-Oxidation of CO: Pt Modified Ru(0001) Electrodes. Surf. Sci. 2015, 631, 248-257.

(10) Ruge, M.; Drnec, J.; Rahn, B.; Reikowski, F.; Harrington, D. A.; Carlà, F.; Felici, R.; Stettner, J.; Magnussen, O. M. Structural Reorganization of $\operatorname{Pt}(111)$ Electrodes by Electrochemical Oxidation and Reduction. J. Am. Chem. Soc. 2017, 139, 4532-4539.

(11) Wen, R.; Hong, M.; Byon, H. R. In Situ AFM Imaging of $\mathrm{Li}_{-} \mathrm{O}_{2}$ Electrochemical Reaction on Highly Oriented Pyrolytic Graphite with Ether-Based Electrolyte. J. Am. Chem. Soc. 2013, 135, 10870-10876.

(12) Liu, C.; Ye, S. In Situ Atomic Force Microscopy (AFM) Study of Oxygen Reduction Reaction on a Gold Electrode Surface in a Dimethyl Sulfoxide (DMSO)-Based Electrolyte Solution. J. Phys. Chem. C 2016, 120, 25246-25255.

(13) Polcari, D.; Dauphin-Ducharme, P.; Mauzeroll, J. Scanning Electrochemical Microscopy: A Comprehensive Review of Experimental Parameters from 1989 to 2015. Chem. Rev. 2016, 116, 13234-13278.

(14) Bard, A. J.; Fan, F.-R. F.; Pierce, D. T.; Unwin, P. R.; Wipf, D. O.; Zhou, F. Chemical Imaging of Surfaces with the Scanning Electrochemical Microscope. Science (80-. ). 1991, 254, 68-74.

(15) Pfisterer, J. H. K.; Liang, Y.; Schneider, O.; Bandarenka, A. S. Direct Instrumental Identification of Catalytically Active Surface Sites. Nature 2017, 549, 74-77.

(16) Pfisterer, J. H. K.; Baghernejad, M.; Giuzio, G.; Domke, K. F. Reactivity Mapping of Nanoscale Defect Chemistry under Electrochemical Reaction Conditions. Nat. Commun. 2019, 10, 5702.

(17) Macias-Romero, C.; Nahalka, I.; Okur, H. I.; Roke, S. Optical Imaging of Surface Chemistry and Dynamics in Confinement. Science (80-. ). 2017, 357, 784-788.

(18) Macias-Romero, C.; Didier, M. E. P.; Jourdain, P.; Marquet, P.; Magistretti, P.; Tarun, O. B.; Zubkovs, V.; Radenovic, A.; Roke, S. High Throughput Second Harmonic Imaging for Label-Free Biological Applications. Opt. Express 2014, 22, 31102.

(19) Macias-Romero, C.; Didier, M. E. P.; Zubkovs, V.; Delannoy, L.; Dutto, F.; Radenovic, A.; Roke, S. Probing Rotational and Translational Diffusion of Nanodoublers in Living Cells on Microsecond Time Scales. Nano Lett. 2014, 14, 2552-2557.

(20) Macias-Romero, C.; Teulon, C.; Didier, M.; Roke, S. Endogenous SHG and 2PEF Coherence Imaging of Substructures in Neurons in 3D. Opt. Express 2019, 27, 2235.

(21) Horswell, S. L.; Pinheiro, A. L. N.; Savinova, E. R.; Danckwerts, M.; Pettinger, B.; Zei, M.; Ertl, G. A Comparative Study of Hydroxide Adsorption on the (111), (110), and (100) Faces of Silver with Cyclic Voltammetry, Ex Situ Electron Diffraction, and In Situ Second Harmonic Generation. Langmuir 2004, 20, 10970-10981.

(22) Tamburello-Luca, A. A.; Hébert, P.; Brevet, P. F.; Girault, H. H. Surface Second 
Harmonic Study of Anion Adsorption at the Mercury | Electrolyte Interface. J. Electroanal. Chem. 1996, 409, 123-129.

(23) Aktsipetrov, O. A.; Melnikov, A. V.; Murzina, T. V.; Nikulin, A. A.; Rubtsov, A. N. DC-Electric-Field-Induced Optical Second Harmonic Generation at the Smooth MetalElectrolyte Interface. Surf. Sci. 1995, 336, 225-231.

(24) Pettinger, B.; Lipkowski, J.; Mirwald, S.; Friedrich, A. Specific Adsorption at Au(111) Electrodes Studied by Second Harmonic Generation. J. Electroanal. Chem. 1992, 329, 289-311.

(25) Werner, L.; Marlow, F.; Hill, W.; Retter, U. Optical Second Harmonic Generation at Hg Electrodes. The Adsorption of Aniline. Chem. Phys. Lett. 1992, 194, 39-44.

(26) Lüpke, G.; Marowsky, G.; Steinhoff, R.; Friedrich, A.; Pettinger, B.; Kolb, D. M. Symmetry Superposition Studied by Surface Second-Harmonic Generation. Phys. Rev. B 1990, 41, 6913-6919.

(27) Richmond, G. L.; Robinson, J. M.; Shannon, V. L. Second Harmonic Generation Studies of Interfacial Structure and Dynamics. Prog. Surf. Sci. 1988, 28, 1-70.

(28) Richmond, G. L. Surface Second-Harmonic Generation from Sulfate Ions Adsorbed on Silver Electrodes. Chem. Phys. Lett. 1984, 106, 26-29.

(29) Georgiadis, R.; Neff, G. A.; Richmond, G. L. Effect of Optical Resonances on the Second Harmonic Response from $\operatorname{Ag}(111)$ and $\operatorname{Ag}(110)$ in Solution. J. Chem. Phys. 1990, $92,4623-4625$.

(30) Guyot-Sionnest, P.; Tadjeddine, A.; Liebsch, A. Electronic Distribution and Nonlinear Optical Response at the Metal-Electrolyte Interface. Phys. Rev. Lett. 1990, 64, 16781681.

(31) Baten, S. M. A.; Taylor, A. G.; Wilde, C. P. Second Harmonic Generation Studies of the Oxidation of Metal Electrodes: Compact and Hydrous Oxide Growth at Gold Electrodes in Acid Solutions. Electrochim. Acta 2008, 53, 6829-6834.

(32) Shen, Y. R. Optical Second Harmonic Generation at Interfaces. Annu. Rev. Phys. Chem. 1989, 40, 327-350.

(33) Guyot-Sionnest, P.; Tadjeddine, A. Study of $\mathrm{Ag}(111)$ and $\mathrm{Au}(111)$ Electrodes by Optical Second-harmonic Generation. J. Chem. Phys. 1990, 92, 734-738.

(34) Pettinger, B.; Lipkowski, J.; Mirwald, S. In Situ SHG Studies of Adsorption Induced Surface Reconstruction of $\mathrm{Au}(111)$-Electrodes. Electrochim. Acta 1995, 40, 133-142.

(35) Pettinger, B.; Mirwald, S.; Lipkowski, J. Microfacetting of Au(110) Electrodes: An Optical Second-Harmonic Generation Study. Appl. Phys. A Mater. Sci. Process. 1995, 60, 121-125.

(36) Pettinger, B.; Mirwald, S.; Lipkowski, J. In-Situ SHG Studies of Ionic and Molecular Adsorption on Surface Structure of $\mathrm{Au}(111)$-Electrodes. Berichte der 
Bunsengesellschaft für Phys. Chemie 1993, 97, 395-398.

(37) Bilger, C.; Pettinger, B. The SHG Anisotropy Pattern of a Reconstructed Gold(110) Electrode. Chem. Phys. Lett. 1998, 294, 425-433.

(38) Tong, Y.; Lapointe, F.; Thämer, M.; Wolf, M.; Campen, R. K. Hydrophobic Water Probed Experimentally at the Gold Electrode/Aqueous Interface. Angew. Chemie Int. Ed. 2017, 56, 4211-4214.

(39) Bloembergen, N.; Chang, R. K.; Jha, S. S.; Lee, C. H. Optical Second-Harmonic Generation in Reflection from Media with Inversion Symmetry. Phys. Rev. 1968, 174, 813-822.

(40) Corn, R. M.; Romagnoli, M.; Levenson, M. D.; Philpott, M. R. Second Harmonic Generation at Thin Film Silver Electrodes via Surface Polaritons. J. Chem. Phys. 1984, 81, 4127-4132.

(41) Richmond, G. L. Characterization of the Silver-Aqueous Electrolyte Interface by Optical Second Harmonic Generation. Langmuir 1986, 2, 132-139.

(42) Gomopoulos, N.; Lütgebaucks, C.; Sun, Q.; Macias-Romero, C.; Roke, S. Label-Free Second Harmonic and Hyper Rayleigh Scattering with High Efficiency. Opt. Express 2013, 21,815 .

(43) Barker, A. S. Optical Measurements of Surface Plasmons in Gold. Phys. Rev. B 1973, 8, 5418-5426.

(44) Diaz-Morales, O.; Calle-Vallejo, F.; de Munck, C.; Koper, M. T. M. Electrochemical Water Splitting by Gold: Evidence for an Oxide Decomposition Mechanism. Chem. Sci. 2013, 4, 2334.

(45) Somorjai, G. A. Modern Surface Science and Surface Technologies: An Introduction. Chem. Rev. 1996, 96, 1223-1236.

(46) Schlögl, R. Heterogeneous Catalysis. Angew. Chemie Int. Ed. 2015, 54, 3465-3520.

(47) Zwaschka, G.; Wolf, M.; Campen, R. K.; Tong, Y. A Microscopic Model of the Electrochemical Vibrational Stark Effect: Understanding VSF Spectroscopy of (Bi)Sulfate on Pt(111). Surf. Sci. 2018, 678, 78-85.

(48) Eggen, P.-O. A Simple Hydrogen Electrode. J. Chem. Educ. 2009, 86, 352.

(49) Gonella, G.; Lütgebaucks, C.; de Beer, A. G. F.; Roke, S. Second Harmonic and SumFrequency Generation from Aqueous Interfaces Is Modulated by Interference. $J$. Phys. Chem. C 2016, 120, 9165-9173.

(50) Lee, C. H.; Chang, R. K.; Bloembergen, N. Nonlinear Electroreflectance in Silicon and Silver. Phys. Rev. Lett. 1967, 18, 167-170.

(51) Wang, F. X.; Rodríguez, F. J.; Albers, W. M.; Ahorinta, R.; Sipe, J. E.; Kauranen, M. Surface and Bulk Contributions to the Second-Order Nonlinear Optical Response of a Gold Film. Phys. Rev. B 2009, 80, 233402. 
(52) Smith, D. D.; Yoon, Y.; Boyd, R. W.; Campbell, J. K.; Baker, L. A.; Crooks, R. M.; George, M. Z -Scan Measurement of the Nonlinear Absorption of a Thin Gold Film. J. Appl. Phys. 1999, 86, 6200-6205.

(53) Rotenberg, N.; Bristow, A. D.; Pfeiffer, M.; Betz, M.; van Driel, H. M. Nonlinear Absorption in Au Films: Role of Thermal Effects. Phys. Rev. B 2007, 75, 155426.

(54) Boyd, R. W.; Shi, Z.; De Leon, I. The Third-Order Nonlinear Optical Susceptibility of Gold. Opt. Commun. 2014, 326, 74-79.

(55) Lee, T. K.; Bristow, A. D.; Hübner, J.; van Driel, H. M. Linear and Nonlinear Optical Properties of Au-Polymer Metallodielectric Bragg Stacks. J. Opt. Soc. Am. B 2006, 23, 2142.

(56) Gold: Science and Applications; Corti, C., Holliday, R., Eds.; CRC Press, 2009.

(57) Niaura, G.; Gaigalas, A. K.; Vilker, V. L. Surface-Enhanced Raman Spectroscopy of Phosphate Anions: Adsorption on Silver, Gold, and Copper Electrodes. J. Phys. Chem. B 1997, 101, 9250-9262.

(58) Conway, B. E.; White, E. R.; Bockris, J. O. Book 1985_Conway - Modern Aspects of Electrochemistry.Pdf; Plenum Press, 1985.

(59) Kasuya, M.; Sogawa, T.; Masuda, T.; Kamijo, T.; Uosaki, K.; Kurihara, K. Anion Adsorption on Gold Electrodes Studied by Electrochemical Surface Forces Measurement. J. Phys. Chem. C 2016, 120, 15986-15992.

(60) Weber, M.; de Moraes, I. R.; Motheo, A. J.; Nart, F. C. In Situ Vibrational Spectroscopy Analysis of Adsorbed Phosphate Species on Gold Single Crystal Electrodes. Colloids Surfaces A Physicochem. Eng. Asp. 1998, 134, 103-111.

(61) Silva, F.; Martins, A. Surface Structural Effects on Specific Adsorption of Oxoanions on Gold Single Crystal Electrodes. J. Electroanal. Chem. 1999, 467, 335-341.

(62) Lopez, N.; Janssens, T. V. W.; Clausen, B. S.; Xu, Y.; Mavrikakis, M.; Bligaard, T.; Nørskov, J. K. On the Origin of the Catalytic Activity of Gold Nanoparticles for LowTemperature CO Oxidation. J. Catal. 2004, 223, 232-235.

(63) Burke, L. D.; Collins, J. A.; Murphy, M. A. Redox and Electrocatalytic Activity of Copper in Base at Unusually Low, Premonolayer Potentials. J. Solid State Electrochem. 1999, 4, 34-41.

(64) Zhumaev, U.; Rudnev, A. V; Li, J.-F.; Kuzume, A.; Vu, T.-H.; Wandlowski, T. ElectroOxidation of $\mathrm{Au}(111)$ in Contact with Aqueous Electrolytes: New Insight from in Situ Vibration Spectroscopy. Electrochim. Acta 2013, 112, 853-863.

(65) Yoshida, K.; Kuzume, A.; Broekmann, P.; Pobelov, I. V.; Wandlowski, T. Reconstruction and Electrochemical Oxidation of $\mathrm{Au}(110)$ Surface in $0.1 \mathrm{M} \mathrm{H}_{2} \mathrm{SO}_{4}$. Electrochim. Acta 2014, 139, 281-288.

(66) Angerstein-Kozlowska, H.; Conway, B. E.; Hamelin, A.; Stoicoviciu, L. Elementary 
Steps of Electrochemical Oxidation of Single-Crystal Planes of Au-I. Chemical Basis of Processes Involving Geometry of Anions and the Electrode Surfaces. Electrochim. Acta 1986, 31, 1051-1061.

(67) Angerstein-Kozlowska, H.; Conway, B. E.; Hamelin, A.; Stoicoviciu, L. Elementary Steps of Electrochemical Oxidation of Single-Crystal Planes of Au Part II. A Chemical and Structural Basis of Oxidation of the (111) Plane. J. Electroanal. Chem. Interfacial Electrochem. 1987, 228, 429-453.

(68) Hamelin, A.; Borkowska, Z.; Stafiej, J. A Double Layer Study of the (210) and (111) Faces of Gold in Aqueous NaBF4 Solutions. J. Electroanal. Chem. Interfacial Electrochem. 1985, 189, 85-97.

(69) Cuesta, A.; Kleinert, M.; Kolb, D. M. The Adsorption of Sulfate and Phosphate on $\mathrm{Au}(111)$ and $\mathrm{Au}(100)$ Electrodes: An in Situ STM Study. Phys. Chem. Chem. Phys. 2000, 2, 5684-5690.

(70) Burke, L. D.; Ahern, A. J.; O’Mullane, A. P. High Energy States of Goldand Their Importance in Electrocatalytic Processes at Surfaces and Interfaces. Gold Bull. 2002, $35,3-10$.

(71) Borkowska, Z.; Slimming, U. Perchlorate Adsorption on Polycrystalline Gold Electrodes in Aqueous Perchloric Acid. J. Electroanal. Chem. Interfacial Electrochem. 1991, 312, 237-244.

(72) Climent, V.; García-Araez, N.; Herrero, E.; Feliu, J. Potential of Zero Total Charge of Platinum Single Crystals: A Local Approach to Stepped Surfaces Vicinal to Pt(111). Russ. J. Electrochem. 2006, 42, 1145-1160.

(73) Lang, X.; Qian, L.; Guan, P.; Zi, J.; Chen, M. Localized Surface Plasmon Resonance of Nanoporous Gold. Appl. Phys. Lett. 2011, 98, 093701.

(74) Rodríguez-Fernández, J.; Funston, A. M.; Pérez-Juste, J.; Álvarez-Puebla, R. A.; LizMarzán, L. M.; Mulvaney, P. The Effect of Surface Roughness on the Plasmonic Response of Individual Sub-Micron Gold Spheres. Phys. Chem. Chem. Phys. 2009, 11, 5909.

\section{TOC Graphic}
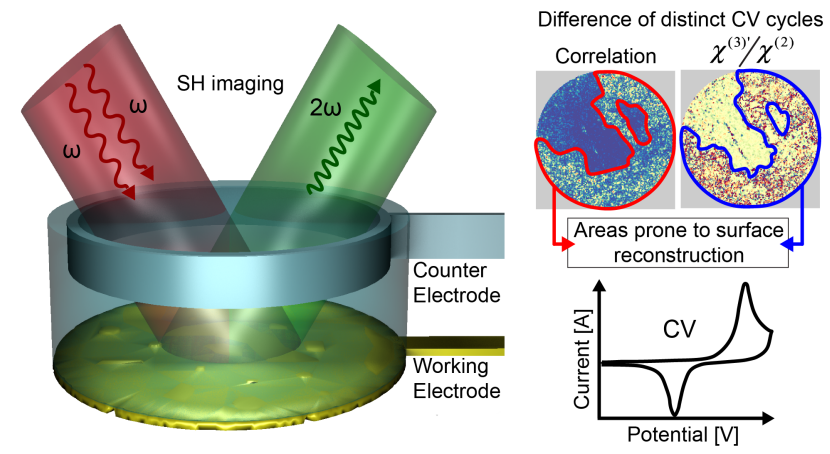